\title{
Optical conductivities and signatures of topological insulators with hexagonal warping
}

\author{
Xiao Xiao and Weijia Wen* \\ Department of Physics, The Hong Kong University of Science and Technology, Clear Water Bay, Kowloon, Hong Kong
}

(Received 16 May 2013; revised manuscript received 8 July 2013; published 30 July 2013)

\begin{abstract}
In this work we study the effect of the hexagonal warping term on the optical conductivities of topological insulators (TIs). The possible optical signatures in the spectra are also discussed in detail. We begin with systematical analyses of the optical conductivities of the TIs by using Kubo's formula. Then, based on the features of the optical conductivities, we study the possible optical signatures due to the warping term. We explore the reflection spectra from a TI thin film growing on a metamaterial. We find that by properly tuning the refraction index of the metamaterial, all features of the warping effect on the conductivities can be detected and identified from the reflection spectra at an experimentally accessible level. Finally, we discuss the possibility of using the Goos-Hänchen effect and the Brewster angle measurement to determine the possible optical signatures of the warping term on the imperfectly quantized optical Hall conductivity.
\end{abstract}

DOI: 10.1103/PhysRevB.88.045442

PACS number(s): 75.70.Tj, 72.20.-i, 78.66.-w, 42.25.Gy

\section{INTRODUCTION}

Topological insulators are bulk insulators which have gapless topologically protected helical surface states. ${ }^{1-8}$ Typically, these surface states are linear in energy dispersion and can be described by the helical Dirac model. ${ }^{1,2,9-11}$ When the time-reversal symmetry of the system is broken by an external field, the interesting magnetoelectric coupling ${ }^{7}$ can be induced by these gapped surface states. The magnetoelectric coupling is the origin of many interesting phenomena proposed recently, such as the possibility of inducing magnetic monopoles, ${ }^{12}$ the giant magneto-optical Kerr effect, ${ }^{13}$ the tunable Casimir effect, ${ }^{14}$ and the enhancement of the spatial coherence of the thermal radiation field. ${ }^{15}$

A recent angle-resolved photoemission experiment for $\mathrm{Bi}_{2} \mathrm{Te}_{3}{ }^{16}$ has revealed that the equal-energy contour would be distorted from the well-known circle shape of the Dirac cone when the equal-energy contour has a large energy difference (i.e., $\sim 0.1 \mathrm{eV}$ ) from the Dirac point. Such geometrical distortion of the equal-energy contour is viewed as the consequence of a crystal and time-reversal-symmetry-constrained hexagonal warping term. ${ }^{11,17}$ Although the transport of the surface states of topological insulators ${ }^{18-26}$ has been studied extensively, only a few authors ${ }^{27-30}$ have discussed the effects of the hexagonal warping on the transport of the surface states. They demonstrated that the warping term would increase the value of the longitudinal conductivity when time-reversal symmetry of the system is protected. Moreover, near the shape change of the equal-energy contour from convex to concave, the resistivity obeys a universal scaling form.

In this paper, we will study the effects of the warping term on the optical properties of topological insulators with and without time-reversal symmetry breaking. Our first step is to calculate the optical conductivities by Kubo's formula. For the optical conductivities contributed by the intraband processes, only the optical longitudinal conductivity is nonvanishing, and we find the value of the conductivity increases with the warping strength. The dissipative conductivities (the real part of the longitudinal conductivity and the imaginary part of the Hall conductivity) contributed by the interband processes relate to a specified equal-energy contour determined by the frequency $\omega$. Consequently, both the surface gap and the warping strength, which can induce the shape change of the equal-energy contour, have an important influence on their values. The dissipationless optical conductivities (the imaginary part of the longitudinal conductivity and the real part of the Hall conductivity) relate to the infinite number of equal-energy contours between $E \in\left[\max \left(\mu_{F}, \Delta\right), \varepsilon_{c}\right]$, where $\mu_{F}$ is the chemical potential, $\Delta$ is the surface gap, and $\varepsilon_{c}$ is the energy cutoff of the surface states. Consequently, the frequency-dependent behaviors of these conductivities for a larger $\max \left(\mu_{F}, \Delta\right)$ would be quite different from those for a smaller $\max \left(\mu_{F}, \Delta\right)$. Based on these features of the optical conductivities, we study the reflection spectra from a thin topological insulator (TI) film growing on a metamaterial, whose refraction index can be tuned in a designed way. ${ }^{31,32}$ We will demonstrate that all features in the optical conductivities can be detected from the optical spectra, as long as the refraction index of the metamaterial is proper. The finite-energy cutoff of the surface states may cause the imperfect quantization of the optical Hall conductivity under the low-frequency incident field. ${ }^{13}$ Therefore, the possibility of using the Goos-Hänchen effect and Brewster angle measurement to detect the warping effect on the imperfectly quantized optical Hall conductivity is also discussed.

\section{MODEL OF SURFACE STATES OF A TOPOLOGICAL INSULATOR WITH WARP EFFECTS}

The surface states of a TI including the hexagonal warping effect under an external magnetic field $B_{z}$ can be modeled by the following Hamiltonian:

$$
\hat{\mathcal{H}}_{0}=v_{F}\left(k_{x} \hat{\sigma}_{y}-k_{y} \hat{\sigma}_{x}\right)+\frac{\gamma}{2}\left(k_{+}^{3}+k_{-}^{3}\right) \hat{\sigma}_{z}+\Delta \sigma_{z},
$$

where $v_{F}$ is the Fermi velocity, $\Delta\left(\propto B_{z}\right)$ characterizes the Zeeman splitting strength due to the time-reversal symmetrybreaking field, $\gamma$ is the hexagonal warping coefficient, $\hat{\sigma}_{i}$ are the Pauli matrices, $k_{x}\left(k_{y}\right)$ is the $x$-component ( $y$-component) momentum of the quasiparticle, and $k_{ \pm}=k_{x} \pm i k_{y}$. We can rewrite $k_{ \pm}=k_{x} \pm i k_{y}=k \exp \left( \pm i \theta_{\vec{k}}\right)$, with $\theta_{\vec{k}}=\cot ^{-1}\left(\frac{k_{x}}{k_{y}}\right)+$ $\pi \Theta\left(-k_{y}\right)$. In the above $\Theta\left(-k_{y}\right)$ is the Heaviside function. By using the definition above, the dispersions of the two bands 


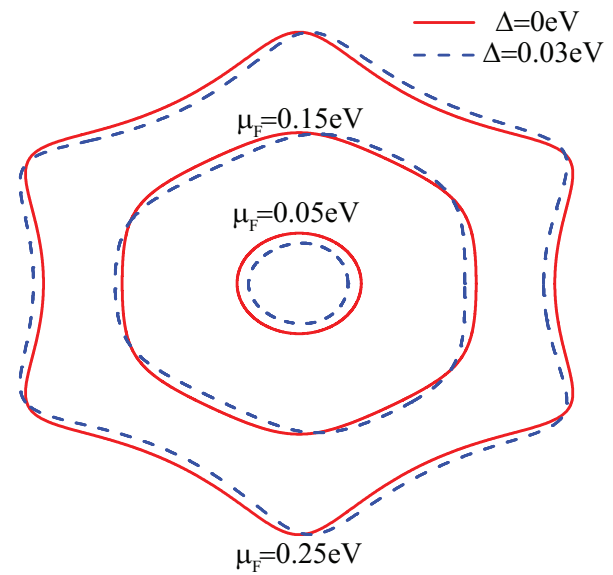

FIG. 1. (Color online) Equal-energy contours at $E=0.05 \mathrm{eV}$ (the smallest one), $E=0.15 \mathrm{eV}$ (the intermediate one), and $E=0.25 \mathrm{eV}$ (the largest one): the red solid curves are for the surface gap $\Delta=0 \mathrm{eV}$ case, and the blue dashed curves are for the surface gap $\Delta=0.03 \mathrm{eV}$ case. It can be seen that the time-reversal symmetry-breaking surface gap would change the rotation symmetry of the equal-energy surface from sixfold to threefold.

can be expressed as

$$
E_{ \pm}= \pm \sqrt{\left(\gamma k^{3} \cos 3 \theta_{\vec{k}}+\Delta\right)^{2}+\left(v_{F} k\right)^{2}}
$$

The time-reversal symmetry-breaking gap $\Delta$ has a very important influence on the shape of the equal-energy contour. This point of view is demonstrated in Fig. 1 by plotting three different equal-energy contours for $\Delta=0$ (red solid curve) and $\Delta \neq 0$ (blue dashed curve). For both the $\Delta=0$ and $\Delta \neq 0$ cases, the shape of the equal-energy contour becomes more and more snowflake-like when the energy difference from the Dirac point increases. For the case $\Delta=0$, the equal-energy contours exhibit sixfold rotation symmetry. However, for the case $\Delta \neq 0$, the equal-energy contours manifest threefold rotation symmetry. The shape change due to the surface gap indicates that the response of the TI to the electromagnetic (EM) radiation would be affected by the surface gap.

The eigenstates of the Hamiltonian can be obtained by rotating the eigenstates of $\hat{\sigma}_{z}$, and we denote them as

$$
\begin{aligned}
& |+, \vec{k}\rangle=\left(\begin{array}{c}
\cos \frac{\beta}{2} \\
i \sin \frac{\beta}{2} \exp \left(i \theta_{\vec{k}}\right)
\end{array}\right) \\
& |-, \vec{k}\rangle=\left(\begin{array}{c}
\sin \frac{\beta}{2} \\
-i \cos \frac{\beta}{2} \exp \left(i \theta_{\vec{k}}\right)
\end{array}\right),
\end{aligned}
$$

where $\beta$ is defined as $\cos \beta=\frac{k^{3} \cos 3 \theta_{\vec{k}}+\Delta}{E_{+}}$. With these preparations, we are ready to consider the response of the surface states to the external EM radiation.

\section{OPTICAL CONDUCTIVITIES}

To describe the responses of the system to the external EM field, we calculate the optical conductivities by using Kubo's formula:

$$
\begin{aligned}
\sigma_{\alpha \beta}\left(\vec{q}, \omega_{n}\right)= & \frac{-i e^{2}}{4 \pi^{2}} \sum_{\vec{k}, \lambda, \lambda^{\prime}}\left(\frac{\partial \mathcal{H}}{\partial k_{\alpha}}\right)_{\lambda, \lambda^{\prime}}\left(\frac{\partial \mathcal{H}}{\partial k_{\beta}}\right)_{\lambda^{\prime}, \lambda} \\
& \times \frac{n_{F}\left(\epsilon_{\vec{k}, \lambda^{\prime}}\right)-n_{F}\left(\epsilon_{\vec{k}-\vec{q}, \lambda}\right)}{\left(\omega_{n}+\epsilon_{\vec{k}, \lambda^{\prime}}-\epsilon_{\vec{k}-\vec{q}, \lambda}\right)\left(\epsilon_{\vec{k}, \lambda^{\prime}}-\epsilon_{\vec{k}-\vec{q}, \lambda}\right)},
\end{aligned}
$$

where $\lambda$ and $\lambda^{\prime}$ denote the band index, $n_{F}$ is the Dirac-Fermi distribution function, and $\left(\frac{\partial \mathcal{H}}{\partial k_{\alpha}}\right)_{\lambda, \lambda^{\prime}}=\left(v_{\alpha}\right)_{\lambda, \lambda^{\prime}}$ is the expectation value of the $\alpha$-component velocity operator. In Eq. (4), $\omega_{n}$ is the Matsubara fermion frequency, and it relates to the real frequency by $\omega_{n} \rightarrow \omega+i /(2 \tau)$, where $\tau$ is the elastic scattering time. Below, we will discuss each component of the optical conductivities separately.

Usually, the model of the surface states should have an energy cutoff because the bulk gap is always finite, which can serve as a natural energy cutoff. In this paper, we set the bulk gap of $\mathrm{Bi}_{2} \mathrm{Te}_{3}\left(\varepsilon_{c}=0.3 \mathrm{eV}\right)^{16}$ as the energy cutoff of the model of the surface states. This means that the frequency we are interested in should be smaller than $2 \varepsilon_{c}=0.6 \mathrm{eV}$, so that we do not have to consider the contributions from the transitions between the bulk conducting band and the valance band. Moreover, we assume the Fermi velocity in the surface model is $2.55 \mathrm{eV} \AA$ (Ref. 16) throughout the study. For convenience of expression, we set the unit of $1 / k$ as $\AA=1$ hereafter. Consequently, the unit of Fermi velocity becomes eV, not $\mathrm{eV} \AA$, and so does the unit of warping strength. For the scattering time, in calculations one can assume that $1 /(2 \tau)$ is proportional to the temperature of the system $T .{ }^{33}$ Since we are interested in the low-temperature case, we use $\tau=1000 / \mathrm{eV}$ throughout.

\section{A. Intraband contributions}

We first consider the intraband contribution to the longitudinal optical conductivity $\sigma_{x x}(\omega)$, and the explicit expression of the optical conductivity is given in units of $e^{2} / h$ by

$$
\sigma_{x x}^{\text {intra }}(\omega)=\frac{-i}{2 \pi \omega} \sum_{\vec{k}}\left|\left(v_{x}\right)_{+,+}\right|^{2} \frac{d n_{F}\left(\varepsilon_{+}\right)}{d \varepsilon_{+}},
$$

where we assume that the Fermi surface crosses the surfaceconducting band, so we can consider only the conducting band $\lambda=\lambda^{\prime}=+$. This is due to the fact that, physically, only the quasiparticles around the Fermi surface contribute to the conductivity. In this paper, we consider only the low-temperature case, which means that $\frac{d n_{F}\left(\varepsilon_{+}\right)}{d \varepsilon_{+}}=-\delta\left(\varepsilon_{+}-\mu_{F}\right)$ is a good approximation. The $\delta$ function also indicates the irrelevance of the valence band. In addition, in Eq. (5) $\left|\left(v_{x}\right)_{+,+}\right|^{2}=$ $\left(3 \gamma k^{2} \cos 2 \theta \cos \beta+v_{F} \sin \beta \cos \theta\right)^{2}$. After changing the integral according to $k$ to the integral according to $\varepsilon_{+}$, we obtain

$$
\begin{aligned}
\sigma_{x x}^{\text {intra }}(\omega)= & \frac{i}{2 \pi \omega} \Theta\left(\mu_{F}-\Delta\right) \\
& \times \int \frac{d \theta \mu_{F}\left|\left(v_{x}\right)_{+,+}\right|^{2}}{3 \gamma\left(\gamma k^{3} \cos 3 \theta+\Delta\right) k \cos 3 \theta+v_{F}^{2}},
\end{aligned}
$$

where we have already integrated the energy $\varepsilon_{+} ; k$ in Eq. (6) is a function of $\varepsilon_{+}$and $\theta$ and can be determined from the dispersion Eq. (2), and $\Theta\left(\mu_{F}-\Delta\right)$ is a step function. As the 

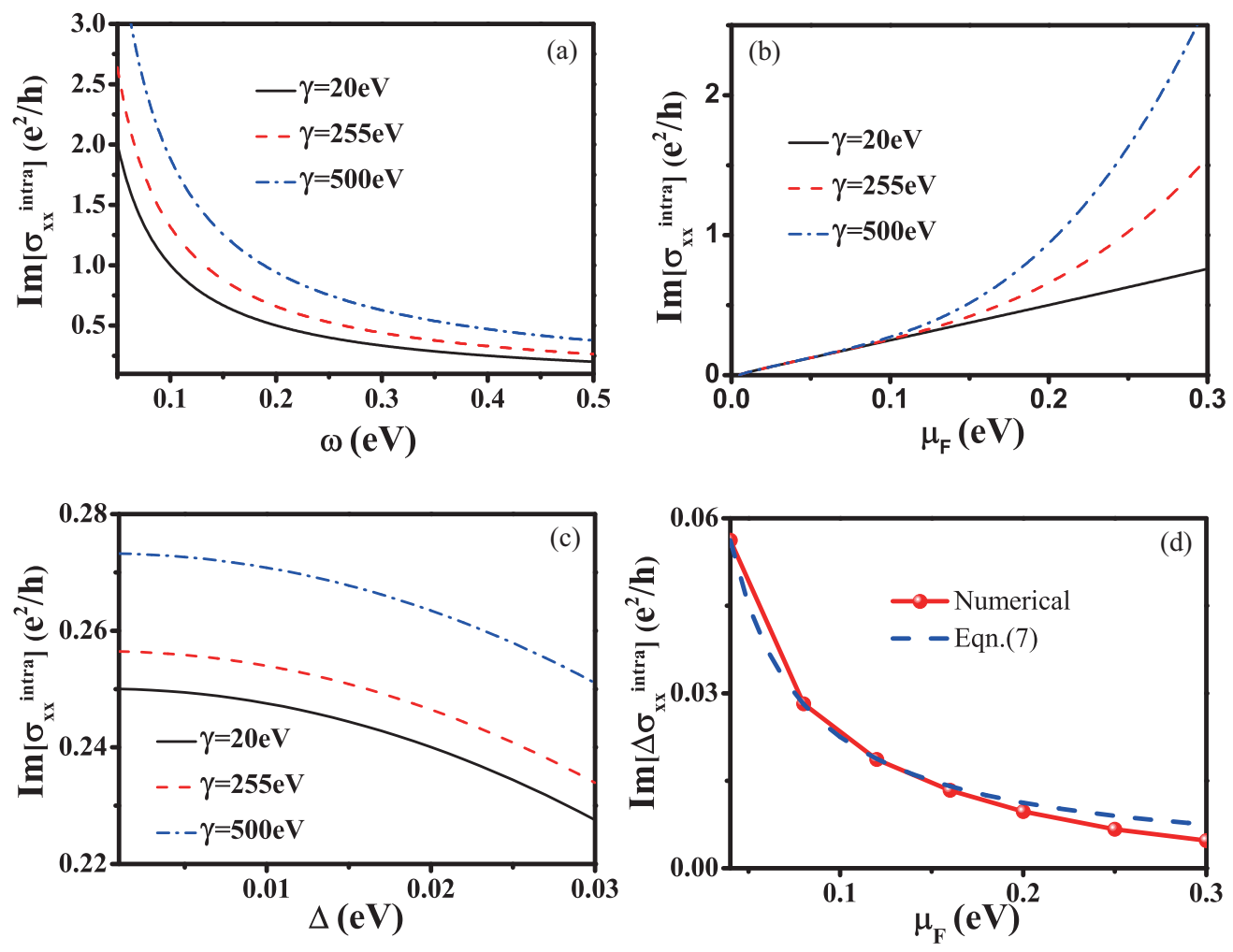

FIG. 2. (Color online) (a) $\operatorname{Im}\left[\sigma_{x x}^{\text {intra }}(\omega)\right]$ as a function of frequency $\omega$ for three different warping strengths. (b) The influence of the position of the chemical potential $\mu_{F}$ on $\operatorname{Im}\left[\sigma_{x x}^{\text {intra }}(\omega=0.2 \mathrm{eV})\right]$ for the three different warping strengths at the frequency $\omega=0.2 \mathrm{eV}$. (c) $\operatorname{Im}\left[\sigma_{x x}^{\text {intra }}(\omega=0.2 \mathrm{eV})\right]$ as a function of the surface gap for three different warping strengths, where we set the chemical potential as $\mu_{F}=0.1 \mathrm{eV}$ and the frequency as $\omega=0.2 \mathrm{eV}$; for all three warping strengths we find that the conductivity decreases with the increment of the surface gap. (d) $\operatorname{Im}\left[\Delta \sigma_{x x}^{\text {intra }}\right]=\operatorname{Im}\left[\sigma_{x x}^{\text {intra }}(\Delta=0.001 \mathrm{eV})\right]-\operatorname{Im}\left[\sigma_{x x}^{\text {intra }}(\Delta=0.03 \mathrm{eV})\right]$ as a function of the chemical potential $\mu_{F}$ : the red curve with symbols is calculated by setting the warping strength as $\gamma=255 \mathrm{eV}$ and the frequency as $\omega=0.2 \mathrm{eV}$, and the blue dashed line is calculated using Eq. (7) (without warping) for $\omega=0.2 \mathrm{eV}$. The quantity $\operatorname{Im}\left[\Delta \sigma_{x x}^{\text {intra }}\right]$ characterizes the difference in the conductivity induced by different surface gaps. This indicates that the influence of the surface gap becomes weak when the chemical potential is much larger than the surface gap.

warping strength is zero, we find

$$
\sigma_{x x}^{\text {intra }}(\omega)=\frac{i}{2} \frac{\left(\mu_{F}^{2}-\Delta^{2}\right)}{\omega \mu_{F}} \Theta\left(\mu_{F}-\Delta\right) .
$$

Thus, the conductivity reduces to the one for a topological insulator under an external magnetic field but with zero warping strength. ${ }^{13,15}$

Similarly, the contribution from the intraband processes to the optical Hall conductivity $\sigma_{x x}(\omega)$ is given by

$$
\sigma_{x y}^{\text {intra }}(\omega)=\frac{-i}{2 \pi \omega} \sum_{\vec{k}}\left(v_{x}\right)_{+,+}\left(v_{y}\right)_{+,+} \frac{d n_{F}\left(\varepsilon_{+}\right)}{d \varepsilon_{+}},
$$

where the product of the expectation values of the velocity operators is given by

$$
\begin{aligned}
\left(v_{x}\right)_{+,+}\left(v_{y}\right)_{+,+}= & -9 \gamma^{2} k^{4} \cos 2 \theta \sin 2 \theta \cos ^{2} \beta \\
& -3 \gamma k^{2} v_{F} \sin \theta \cos \beta \sin \beta \\
& +v_{F}^{2} \sin \theta \cos \theta \sin ^{2} \beta .
\end{aligned}
$$

One may notice that all three terms in Eq. (9) have integer number periodicities in the region $\theta \in[0,2 \pi]$, which implies that the intraband processes have no contribution to the optical Hall conductivity. This point of view is supported by our numerical calculations.
As we have discussed above, the only nonvanishing contribution from the intraband processes is the imaginary part of the optical longitudinal conductivity when the chemical potential $\mu_{F}$ is larger than the surface gap $\Delta$. In Fig. 2(a), we plot $\operatorname{Im}\left[\sigma_{x x}^{\text {intra }}(\omega)\right]$ as the function of the frequency $\omega$ for three different warping strengths, $\gamma=20 \mathrm{eV}, \gamma=255 \mathrm{eV}$, and $\gamma=500 \mathrm{eV}$, where we assume that the surface gap $\Delta$ is $0.005 \mathrm{eV}$ and the chemical potential $\mu_{F}$ is $0.2 \mathrm{eV}$. We notice that the stronger the warping strength is, the larger the conductivity is throughout the frequency regime under consideration. In Fig. 2(b), we show the influence of the position of the chemical potential on $\operatorname{Im}\left[\sigma_{x x}^{\text {intra }}(\omega=0.2 \mathrm{eV})\right]$, where we assume the frequency $\omega$ and the surface gap $\Delta$ to be 0.2 and $0.005 \mathrm{eV}$, respectively. The results indicate that only when the chemical potential has a larger energy difference than the Dirac point can the obvious consequences of the warping effect be observed from $\operatorname{Im}\left[\sigma_{x x}^{\text {intra }}(\omega)\right]$. Further, in Fig. 2(c) we show the influence of the surface gap on $\operatorname{Im}\left[\sigma_{x x}^{\text {intra }}(\omega=0.2 \mathrm{eV})\right]$ for three different warping strengths, $\gamma=20 \mathrm{eV}, \gamma=255 \mathrm{eV}$, and $\gamma=500 \mathrm{eV}$. In the calculation, we have assumed that the chemical potential is $\mu_{F}=0.1 \mathrm{eV}$ and the frequency is $\omega=0.2 \mathrm{eV}$. From Fig. 2(c), we notice that for all three warping strengths the value of $\operatorname{Im}\left[\sigma_{x x}^{\text {intra }}(\omega=0.2 \mathrm{eV})\right]$ decreases with the increment of the surface gap. The change in the conductivity can be 
characterized by the quantity $\operatorname{Im}\left[\Delta \sigma_{x x}^{\text {intra }}\right]=\operatorname{Im}\left[\sigma_{x x}^{\text {intra }}(\Delta=\right.$ $0.001 \mathrm{eV})]-\operatorname{Im}\left[\sigma_{x x}^{\text {intra }}(\Delta=0.03 \mathrm{eV})\right]$, which measures the difference between the conductivity at $\Delta=0.001 \mathrm{eV}$ and that at $\Delta=0.03 \mathrm{eV}$. This quantity is shown as a function of the chemical potential in Fig. 2(d) for $\gamma=255 \mathrm{eV}$ (red curve with symbols). As a comparison, we also calculate the quantity $\operatorname{Im}\left[\Delta \sigma_{x x}^{\text {intra }}\right]$ by using Eq. (7) for the case $\gamma=0 \mathrm{eV}$ (blue dashed curve). The results in Fig. 2(d) illustrate that the influence of the surface gap on $\operatorname{Im}\left[\sigma_{x x}^{\text {intra }}(\omega)\right]$ would not be changed much with the variation of the warping strength. All the analyses above indicate that the warping effect can have an influence on the imaginary part of the optical longitudinal conductivity through the intraband processes, and these effects can introduce observable differences when the chemical potential and the warping strength are large enough. For the real material $\mathrm{Bi}_{2} \mathrm{Te}_{3}$, the warping strength is $255 \mathrm{eV},{ }^{11,16}$ which is one of the cases studied here; large differences in the imaginary part of the optical longitudinal conductivity due to the intraband processes should be measured by tuning the chemical potential to a large value (i.e., $\mu_{F}>0.1 \mathrm{eV}$ ).

\section{B. Interband contributions}

In this section, let us first consider the optical longitudinal conductivity due to the interband processes. Following Eq. (4), the explicit expression of the conductivity is given by

$$
\begin{aligned}
\sigma_{x x}^{\text {inter }}\left(\omega_{n}\right)= & \frac{i e^{2}}{4 \pi^{2}} \sum_{\vec{k}}\left(v_{x}\right)_{+,-}\left(v_{x}\right)_{-,+} \\
& \times \frac{g\left(\varepsilon_{+}\right)}{2 \varepsilon_{+}}\left(\frac{1}{\omega_{n}+2 \varepsilon_{+}}+\frac{1}{\omega_{n}-2 \varepsilon_{+}}\right),
\end{aligned}
$$

where $g\left(\varepsilon_{+}\right)=n_{F}\left(-\varepsilon_{+}\right)-n_{F}\left(\varepsilon_{+}\right)$. At low temperature, we notice $g\left(\varepsilon_{+}\right)=\Theta\left(\varepsilon_{+}-\mu_{F}\right)$. On the other hand, we notice that $\frac{1}{\omega_{n}+2 \varepsilon_{+}}$has no singularities as $\tau \rightarrow \infty$, so we would have $\frac{1}{\omega_{n}+2 \varepsilon_{+}}=\frac{1}{\omega+2 \varepsilon_{+}}-i \pi \delta\left(\omega+2 \varepsilon_{+}\right)$. However, for $\frac{1}{\omega_{n}-2 \varepsilon_{+}}$, a singularity can be found at $\omega=2 \varepsilon_{+}$, and we take the following approximation to remove the singularity in the calculation: $\frac{1}{\omega_{n}-2 \varepsilon_{+}}=\frac{\omega-2 \varepsilon_{+}}{\left(\omega-2 \varepsilon_{+}\right)^{2}+4 / \tau^{2}}-i \pi \delta\left(\omega-2 \varepsilon_{+}\right)$.

Therefore, the parts containing the $\delta$ function give the contribution to the real part of the optical longitudinal conductivity, and the left parts contribute to the imaginary part of the optical longitudinal conductivity. We write their expression explicitly in units of $e^{2} / h$ :

$$
\begin{aligned}
\operatorname{Re}\left[\sigma_{x x}^{\text {inter }}(\omega)\right]= & \frac{1}{8} \int \frac{d \theta\left|\left(v_{x}\right)_{+,-}\right|^{2} \Theta\left(\omega-2 \max \left(\Delta, \mu_{F}\right)\right)}{3 \gamma\left(\gamma k^{3} \cos 3 \theta+\Delta\right) k \cos 3 \theta+v_{F}^{2}}, \\
\operatorname{Im}\left[\sigma_{x x}^{\text {inter }}(\omega)\right]= & \frac{1}{4 \pi} \iint \frac{d \theta d \varepsilon\left|\left(v_{x}\right)_{+,-}\right|^{2}}{3 \gamma\left(\gamma k^{3} \cos 3 \theta+\Delta\right) k \cos 3 \theta+v_{F}^{2}} \\
& \times\left[\frac{1}{\omega+2 \varepsilon}+\frac{\omega-2 \varepsilon}{(\omega-2 \varepsilon)^{2}+4 / \tau^{2}}\right] \\
& \times \Theta\left(\varepsilon-\mu_{F}\right),
\end{aligned}
$$

where the product of expectations of the velocity operators is given by

$$
\begin{aligned}
\left|\left(v_{x}\right)_{+,-}\right|^{2}= & 9 \gamma^{2} k^{4} \cos ^{2} 2 \theta \sin ^{2} \beta+v_{F}^{2} \sin ^{2} \theta \\
& -6 \gamma k^{2} v_{F} \cos \theta \cos 2 \theta \cos \beta \sin \beta \\
& +v_{F}^{2} \cos ^{2} \theta \cos ^{2} \beta .
\end{aligned}
$$

$k$ in the integral (11a) is determined by the equation $\left(\gamma k^{3} \cos 3 \theta_{\vec{k}}+\Delta\right)^{2}+\left(v_{F} k\right)^{2}=\omega^{2} / 4$, and $k$ in $(11 \mathrm{~b})$ is given by $\left(\gamma k^{3} \cos 3 \theta_{\vec{k}}+\Delta\right)^{2}+\left(v_{F} k\right)^{2}=\varepsilon^{2}$. When the warping strength tends to zero, we notice that

$$
\begin{aligned}
& \operatorname{Re}\left[\sigma_{x x}^{\text {inter }}(\omega)\right]=\left[\frac{\pi}{8}+2 \pi\left(\frac{\Delta}{2 \omega}\right)^{2}\right] \Theta\left(\omega-2 \max \left(\Delta, \mu_{F}\right)\right), \\
& \operatorname{Im}\left[\sigma_{x x}^{\text {inter }}(\omega)\right]=\frac{1}{4}\left(\begin{array}{c}
-2 \frac{\Delta^{2}}{\omega}\left[\frac{1}{\varepsilon_{c}}-\frac{1}{\max \left(\Delta, \mu_{F}\right)}\right] \\
+\frac{1}{2}\left[1+4\left(\frac{\Delta}{\omega}\right)^{2}\right] f(\omega)
\end{array}\right)
\end{aligned}
$$

where $f(\omega)=\ln \left|\left(\omega+2 \varepsilon_{c}\right) /\left(\omega-2 \varepsilon_{c}\right)\right|-\ln \mid[\omega+$ $\left.2 \max \left(\Delta, \mu_{F}\right)\right] /\left[\omega-2 \max \left(\Delta, \mu_{F}\right)\right] \mid$. These results exactly reduce to the conductivities without the warping term. ${ }^{13,15}$

The contribution of the interband processes to the optical Hall conductivity has a similar mathematical structure, and we can obtain their explicit expressions by following similar procedures as above. However, one may have to pay attention to the product of expectations of the velocity operators:

$$
\begin{aligned}
\left(v_{x}\right)_{+,-} & \left(v_{y}\right)_{-,+} \\
= & -9 \gamma^{2} k^{4} \cos 2 \theta \sin 2 \theta \sin ^{2} \beta-v_{F}^{2} \sin \theta \cos \theta \\
& +3 \gamma k^{2} v_{F} \sin \theta \cos \beta \sin \beta+v_{F}^{2} \cos \theta \sin \theta \cos ^{2} \beta \\
& -i 3 v_{F} \gamma k^{2} \sin \beta \cos 3 \theta+i v_{F}^{2} \cos \beta
\end{aligned}
$$

which is no longer real but complex. One may further notice that the real part of the product $\left(v_{x}\right)_{+,-}\left(v_{y}\right)_{-,+}$has an integer number of periodicities for $\theta \in[0,2 \pi]$, which means that the terms containing the real part of $\left(v_{x}\right)_{+,-}\left(v_{y}\right)_{-,+}$simply vanish after the integration according to $\theta$. Given these facts, the expression of the optical Hall conductivity due to the interband processes is given by

$$
\begin{aligned}
\operatorname{Re} & {\left[\sigma_{x y}^{\text {inter }}(\omega)\right] } \\
= & \frac{1}{4 \pi} \iint \frac{d \theta d \varepsilon \operatorname{Im}\left[\left(v_{x}\right)_{+,-}\left(v_{y}\right)_{-,+}\right]}{3 \gamma\left(\gamma k^{3} \cos 3 \theta+\Delta\right) k \cos 3 \theta+v_{F}^{2}} \\
& \times\left[\frac{1}{\omega+2 \varepsilon}-\frac{\omega-2 \varepsilon}{(\omega-2 \varepsilon)^{2}+4 / \tau^{2}}\right] \Theta\left(\varepsilon-\mu_{F}\right), \\
\operatorname{Im} & {\left[\sigma_{x y}^{\text {inter }}(\omega)\right] } \\
= & \frac{1}{8} \int \frac{d \theta \operatorname{Im}\left[\left(v_{x}\right)_{+,-}\left(v_{y}\right)_{-,+}\right] \Theta\left(\omega-2 \max \left(\Delta, \mu_{F}\right)\right)}{3 \gamma\left(\gamma k^{3} \cos 3 \theta+\Delta\right) k \cos 3 \theta+v_{F}^{2}} .
\end{aligned}
$$

By considering the zero-warping limit $(\gamma \rightarrow 0)$, we can recover the results for a TI with no warping effect: ${ }^{13,15}$

$$
\begin{aligned}
& \operatorname{Re}\left[\sigma_{x y}^{\text {inter }}(\omega)\right]=-\Delta /(2 \omega) f(\omega), \\
& \operatorname{Im}\left[\sigma_{x y}^{\text {inter }}(\omega)\right]=\pi \Delta /(2 \omega) \Theta(\omega-2 \max (\Delta, \mu)) .
\end{aligned}
$$

In Fig. 3(a), by integrating Eq. (11a) exactly we first show the effects of the surface gap $\Delta$ on the real part of the optical longitudinal conductivity $\operatorname{Re}\left[\sigma_{x x}^{\text {inter }}(\omega)\right]$ due to the interband processes. In the calculation, we set the warping strength as $255 \mathrm{eV}$ (the warping strength of $\mathrm{Bi}_{2} \mathrm{Te}_{3}$ ) and the chemical potential as $0.05 \mathrm{eV}$. From the results, we notice that with the increment of the surface gap $\Delta$, the value of $\operatorname{Re}\left[\sigma_{x x}^{\text {inter }}(\omega)\right]$ increases. In the inset of Fig. 3(a), we show $\operatorname{Re}\left[\sigma_{x x}^{\text {inter }}(\omega)\right]$ for two different surface gaps $(\Delta=0.015 \mathrm{eV}$ and $\Delta=0.03 \mathrm{eV})$, where we set the chemical potential as $0.005 \mathrm{eV}$. Due to the 

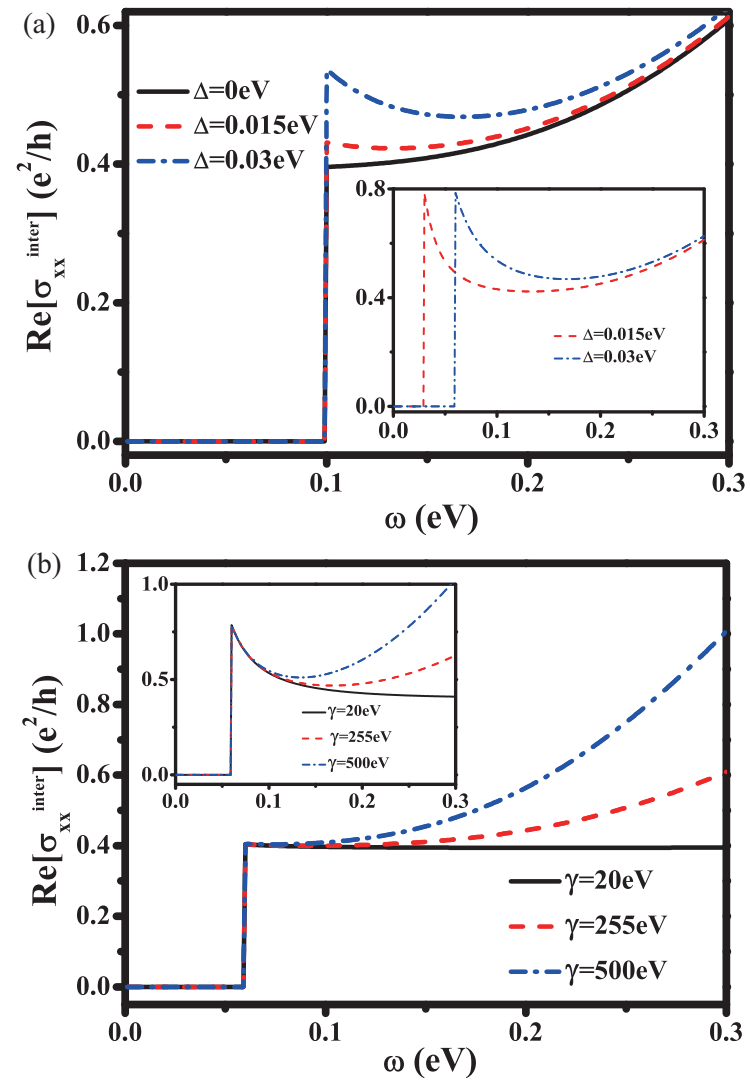

FIG. 3. (Color online) (a) $\operatorname{Re}\left[\sigma_{x x}^{\text {inter }}(\omega)\right]$ as a function of frequency $\omega$ for three different surface gaps, where we set the chemical potential as $\mu_{F}=0.05 \mathrm{eV}$ and the warping strength as $\gamma=255 \mathrm{eV}$. The inset shows $\operatorname{Re}\left[\sigma_{x x}^{\text {inter }}(\omega)\right]$ for two different surface gaps $(\Delta=0.015 \mathrm{eV}$ and $\Delta=0.03 \mathrm{eV})$, which are larger than the chemical potential $\left(\mu_{F}=0.005 \mathrm{eV}\right)$. (b) $\operatorname{Re}\left[\sigma_{x x}^{\text {inter }}(\omega)\right]$ as a function of frequency $\omega$ for three different warping strengths when the chemical potential $\left(\mu_{F}=0.03 \mathrm{eV}\right)$ is larger than the surface gap $(\Delta=0.005 \mathrm{eV})$; the inset shows $\operatorname{Re}\left[\sigma_{x x}^{\text {inter }}(\omega)\right]$ for the same warping strengths when the surface gap $(\Delta=0.03 \mathrm{eV})$ is larger than the chemical potential $\left(\mu_{F}=0.005 \mathrm{eV}\right)$.

step function in Eq. (11), the onsets are observed at 0.03 and $0.06 \mathrm{eV}$ for $\Delta=0.015 \mathrm{eV}$ and $\Delta=0.03 \mathrm{eV}$, respectively. At these two onset energies, the warping does not change the equal-energy surface very much, so we may ignore the warping effect at the two onset energies. Thus, as expected, $\operatorname{Re}\left[\sigma_{x x}^{\text {inter }}(\omega)\right]$ at the two onset energies have almost the same value $\pi / 4\left(e^{2} / h\right)$ [from Eq. 12(a)]. Then from Eq. (12), we find that $\operatorname{Re}\left[\sigma_{x x}^{\text {inter }}(\omega)\right]$ should decrease with the increment of the frequency, until the frequency is large enough to induce much stronger warping.

Next, we study the effects of warping strengths on $\operatorname{Re}\left[\sigma_{x x}^{\text {inter }}(\omega)\right]$. In Fig. 3(b), we plot $\operatorname{Re}\left[\sigma_{x x}^{\text {inter }}(\omega)\right]$ for three different warping strengths, where we assume that the chemical potential is $0.03 \mathrm{eV}$ and the surface gap is $0.005 \mathrm{eV}$. Due to the small surface gap, we recover the universal background (plateau) that is well known in graphene literature ${ }^{30}$ for the weak warping strength (the black curve). With the increment of the warping strength, the value of $\operatorname{Re}\left[\sigma_{x x}^{\text {inter }}(\omega)\right]$ increases much in the regime where frequency $\omega$ is large enough. In the inset, we plot $\operatorname{Re}\left[\sigma_{x x}^{\text {inter }}(\omega)\right]$ for the same warping strengths but with
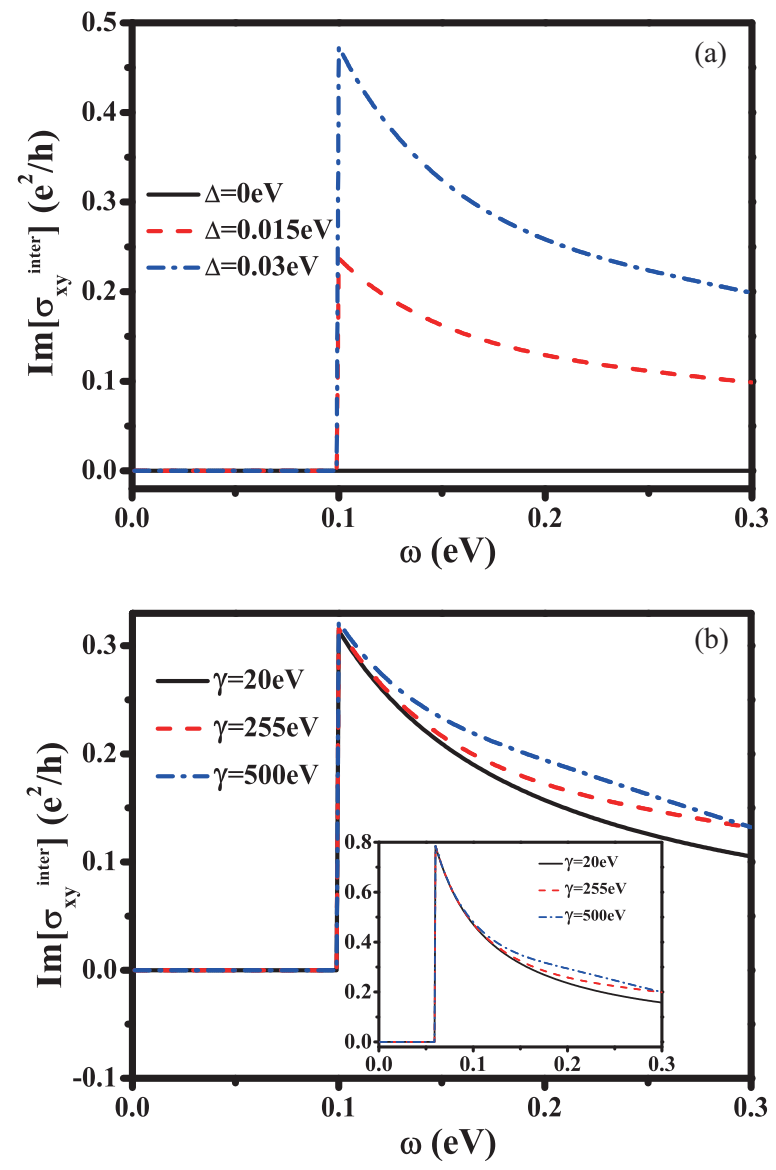

FIG. 4. (Color online) (a) $\operatorname{Im}\left[\sigma_{x y}^{\text {inter }}(\omega)\right]$ as a function of frequency $\omega$ for three different surface gaps, where the chemical potential and the warping strength are set as $\mu_{F}=0.05 \mathrm{eV}$ and $\gamma=255 \mathrm{eV}$ respectively. (b) $\operatorname{Im}\left[\sigma_{x y}^{\text {inter }}(\omega)\right]$ as a function of frequency $\omega$ for three different warping strengths, where we set the chemical potential as $\mu_{F}=0.05 \mathrm{eV}$ and the surface gap as $\Delta=0.02 \mathrm{eV}$. The inset shows $\operatorname{Im}\left[\sigma_{x y}^{\text {inter }}(\omega)\right]$ for the same three warping strengths when setting the chemical potential as $\mu_{F}=0.005 \mathrm{eV}$ and the surface gap as $\Delta=0.03 \mathrm{eV}$.

a much larger surface gap $(\Delta=0.03 \mathrm{eV})$ and much smaller chemical potential $\left(\mu_{F}=0.005 \mathrm{eV}\right)$. The warping effect also shows up clearly when the frequency is large enough.

Similarly, the surface gap also influences the imaginary part of the optical Hall conductivity $\operatorname{Im}\left[\sigma_{x y}^{\text {inter }}(\omega)\right]$ induced by the interband processes. By setting the chemical potential as $\mu_{F}=0.05 \mathrm{eV}$ and the warping strength as $\gamma=255 \mathrm{eV}$, we calculate $\operatorname{Im}\left[\sigma_{x y}^{\text {inter }}(\omega)\right]$ for three different surface gaps. The results are shown in Fig. 4(a). When the surface gap is zero, the conductivity simply vanishes, which coincides with the case with no warping [Eq. (16b)]. With the increment of the surface gap, the conductivity at the onset frequency $\omega=2 \mu_{F}$ becomes larger and larger. For both nonzero gap cases, the conductivities decrease with the frequency when the frequency is larger than the onset frequency. Further, we study the warping effect on $\operatorname{Im}\left[\sigma_{x y}^{\text {inter }}(\omega)\right]$ in Fig. 4(b). In the calculation, we set the chemical potential as $\mu_{F}=0.05 \mathrm{eV}$, which is larger than the surface gap $\Delta=0.02$. For the completeness, in the inset we plot $\operatorname{Im}\left[\sigma_{x y}^{\text {inter }}(\omega)\right]$ for the same three warping strengths under the condition that the surface gap $(\Delta=0.03 \mathrm{eV})$ is 
much larger than the chemical potential $\left(\mu_{F}=0.005 \mathrm{eV}\right)$. For both cases, the increments of the conductivity due to the warping effect are observed clearly when the frequency is large enough. From Eq. (16b), one can expect the maximal value of the conductivity occurring at $\omega=2 \max (\Delta, \mu)$, which are exactly the onset energies in Fig. 4. For the equal-energy surface around $\omega=0.06 \mathrm{eV}$ (see Fig. 1), the warping effect is not so strong, so we observe in the inset almost the same maximal value $\pi \Delta /(2 \omega)=\pi / 4 \sim 0.786$ for the three different warping strengths.

Before we discuss the imaginary part of the longitudinal conductivity $\operatorname{Im}\left[\sigma_{x x}^{\text {inter }}(\omega)\right]$ due to the interband processes, we would like to emphasize the difference between $\operatorname{Im}\left[\sigma_{x x}^{\text {inter }}(\omega)\right]$ and its real part. From Eq. (11), we notice that the real part is only relevant with an equal-energy surface, but the imaginary part relates to an infinite number of equal-energy surfaces in the surface-conducting band, whose energies are in between $\max \left(\Delta, \mu_{F}\right)$ and the cutoff energy of the model $\varepsilon_{c}$. The zero-warping limit of $\operatorname{Im}\left[\sigma_{x x}^{\text {inter }}(\omega)\right]$ is given by Eq. (13b). From this equation, we notice that whether the chemical potential is larger or the surface gap is larger, the frequency-dependent behavior of $\operatorname{Im}\left[\sigma_{x x}^{\text {inter }}(\omega)\right]$ is qualitatively similar, although they are different quantitatively. From this point of view, the frequency-dependent behavior of $\operatorname{Im}\left[\sigma_{x x}^{\text {inter }}(\omega)\right]$ should be more relevant to $\max \left(\Delta, \mu_{F}\right)$ (one of the integral limits).

By integrating (11b) numerically, we can study the imaginary part of the longitudinal conductivity due to the interband processes. In Fig. 5(a), we present $\operatorname{Im}\left[\sigma_{x x}^{\text {inter }}(\omega)\right]$ for three different warping strengths by assuming the chemical potential as $\mu_{F}=0.1 \mathrm{eV}$ and the surface gap as $\Delta=0.01 \mathrm{eV}$, which means $\max \left(\Delta, \mu_{F}\right)=0.1 \mathrm{eV}$. In Fig. 5(b), we show the conductivity for the case $\max \left(\Delta, \mu_{F}\right)=0.03 \mathrm{eV}$ with the chemical potential as $\mu_{F}=0.01 \mathrm{eV}$ and the surface gap as $\Delta=0.03 \mathrm{eV}$. From these results, we notice that when $\max \left(\Delta, \mu_{F}\right)$ is large, the obvious difference induced by the warping can be identified, even when the frequency is relatively low. In contrast, for the case shown in Fig. 5(b), where $\max \left(\Delta, \mu_{F}\right)$ is much smaller than that of the case in Fig. 5(a), the difference induced by the warping is tiny when the frequency is small. The second notable difference is that the frequency-dependent behavior of $\operatorname{Im}\left[\sigma_{x x}^{\text {inter }}(\omega)\right]$ is easy to change with the warping effects when $\max \left(\Delta, \mu_{F}\right)$ is smaller: from Fig. 5(b), for the weak warping strength $\gamma=20 \mathrm{eV}$, the conductivity increases all the way after it reaches its minimal at $\omega=2 \Delta$, but for the stronger warping strengths, i.e., $\gamma=255 \mathrm{eV}$ and $\gamma=500 \mathrm{eV}, \operatorname{Im}\left[\sigma_{x x}^{\text {inter }}(\omega)\right]$ decreases with frequency in the regime $\omega \in[0.1 \mathrm{eV}, 0.3 \mathrm{eV}]$. However, such a change of the frequency-dependent behavior of $\operatorname{Im}\left[\sigma_{x x}^{\text {inter }}(\omega)\right]$ is not obvious in Fig. 5(a).

We have to note that the upturns of the conductivities shown in Fig. 5 around $\omega=2 \varepsilon_{c}=0.6 \mathrm{eV}$ may be artifacts due to the energy cutoff of the surface model. This may be clear by looking at Eqs. (11b) and (15a), which contain a term $\frac{\omega-2 \varepsilon}{(\omega-2 \varepsilon)^{2}+4 / \tau^{2}}$. Since $4 / \tau^{2}$ is small, when $\omega$ approaches $2 \varepsilon_{c}$, the value of the conductivity will increase dramatically. However, we would like to emphasize that different warping strengths should still introduce differences in the conductivities in this frequency regime.

The real part of the optical Hall conductivity $\operatorname{Re}\left[\sigma_{x y}^{\text {inter }}(\omega)\right]$ due to the interband processes is then studied by numerically evaluating Eq. (15a). Due to the same argument as for
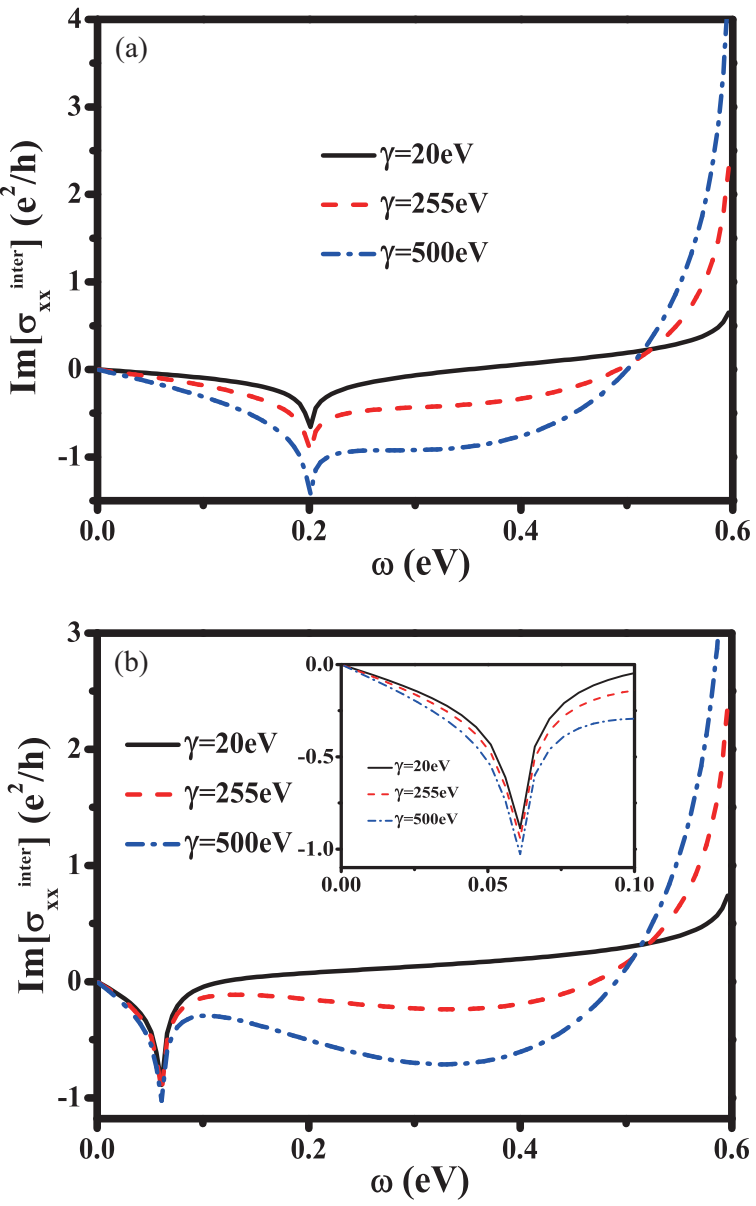

FIG. 5. (Color online) (a) $\operatorname{Im}\left[\sigma_{x x}^{\text {inter }}(\omega)\right]$ as a function of frequency $\omega$ for three different warping strengths, where we set the chemical potential as $\mu_{F}=0.1 \mathrm{eV}$ and the surface gap as $\Delta=0.01 \mathrm{eV}$. (b) $\operatorname{Im}\left[\sigma_{x x}^{\text {inter }}(\omega)\right]$ as a function of frequency $\omega$ for three different warping strengths by setting the chemical potential as $\mu_{F}=0.01 \mathrm{eV}$ and the surface gap as $\Delta=0.03 \mathrm{eV}$. A close-up showing $\operatorname{Im}\left[\sigma_{x y}^{\text {inter }}(\omega)\right]$ in the regime $\omega \in[0 \mathrm{eV}, 0.1 \mathrm{eV}]$ is presented in the inset.

$\operatorname{Im}\left[\sigma_{x x}^{\text {inter }}(\omega)\right]$, the more relevant quantity to the frequencydependent behavior of the conductivity is $\max \left(\Delta, \mu_{F}\right)$. In Fig. $6(a), \operatorname{Re}\left[\sigma_{x y}^{\text {inter }}(\omega)\right]$ is shown as a function of frequency for three different warping strengths by setting the chemical potential as $\mu_{F}=0.1 \mathrm{eV}$ and the surface gap as $\Delta=0.01 \mathrm{eV}$. We also show the frequency-dependent $\operatorname{Re}\left[\sigma_{x y}^{\operatorname{inter}}(\omega)\right]$ for a smaller $\max \left(\Delta, \mu_{F}\right)=0.03 \mathrm{eV}$ in Fig. 6(b), where the chemical potential is $\mu_{F}=0.01 \mathrm{eV}$ and the surface gap is $\Delta=$ $0.03 \mathrm{eV}$. For both cases, the frequency-dependent behaviors of $\operatorname{Re}\left[\sigma_{x y}^{\text {inter }}(\omega)\right]$ are very similar qualitatively when the frequency is large [i.e., $\omega \geqslant 2 \max \left(\Delta, \mu_{F}\right)$ ]. At low frequency, we find that for larger $\max \left(\Delta, \mu_{F}\right)$, there is a maximum for the conductivity $\left(\operatorname{Re}\left[\sigma_{x y}^{\text {inter }}(\omega=0.001 \mathrm{eV})\right]\right)$ at an intermediate warping strength. However, the conductivity $\left(\operatorname{Re}\left[\sigma_{x y}^{\text {inter }}(\omega=\right.\right.$ $0.001 \mathrm{eV})]$ ) will increase with the warping strength all the way for a small $\max \left(\Delta, \mu_{F}\right)$. These points are shown in the insets of Figs. 6(a) and 6(b), respectively. Particularly, when the surface gap is much larger than the frequency and the chemical potential is in the gap, we recover the well-known half-integer quantized optical Hall conductivity. The small deviation observed here is due to the finite-energy cutoff of the model. 

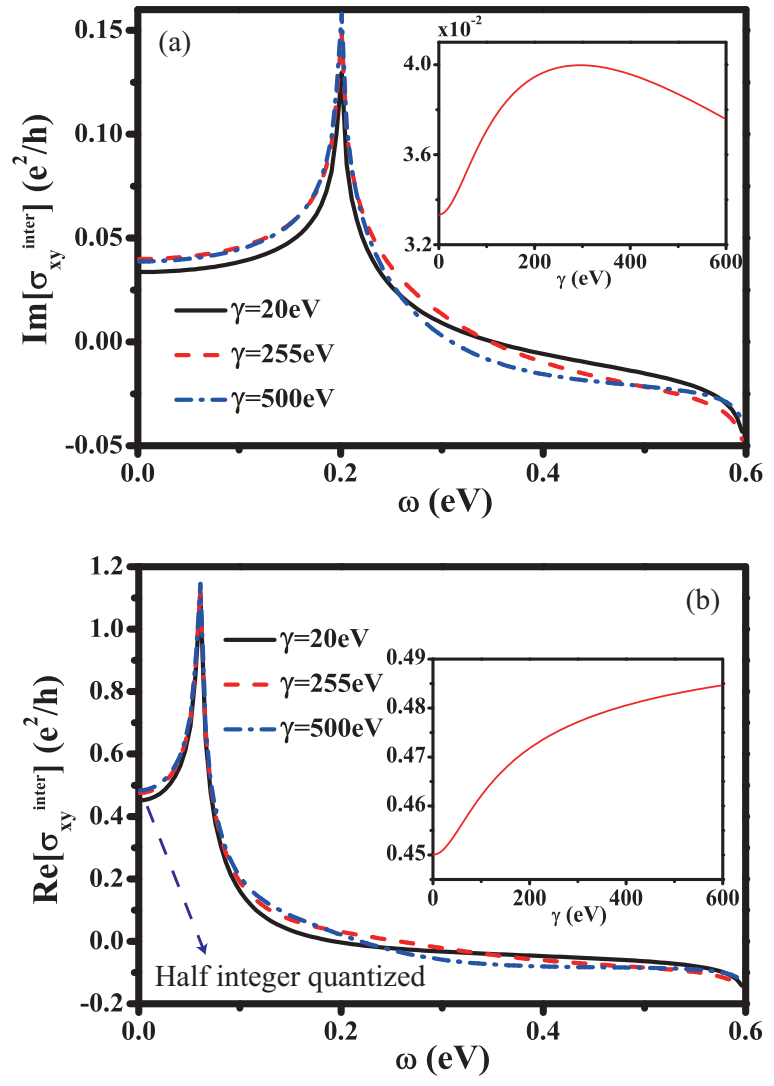

FIG. 6. (Color online) (a) $\operatorname{Re}\left[\sigma_{x y}^{\text {inter }}(\omega)\right]$ as a function of frequency $\omega$ for three different warping strengths, where we set the chemical potential as $\mu_{F}=0.1 \mathrm{eV}$ and the surface gap as $\Delta=0.01 \mathrm{eV}$ [with $\max \left(\Delta, \mu_{F}\right)=0.1 \mathrm{eV}$ ]; the inset shows the influence of the warping strength on the conductivity for the frequency $\omega=0.001 \mathrm{eV}$. (b) $\operatorname{Im}\left[\sigma_{x x}^{\text {inter }}(\omega)\right]$ as a function of frequency $\omega$ for three different warping strengths by setting the chemical potential as $\mu_{F}=0.01 \mathrm{eV}$ and the surface gap as $\Delta=0.03 \mathrm{eV}$ [with $\max \left(\Delta, \mu_{F}\right)=0.03 \mathrm{eV}$ ]; the warping strength dependent on the conductivity for $\omega=0.001$ $\mathrm{eV}$ is shown in the inset.

\section{OPTICAL SIGNATURES}

\section{A. Reflection coefficients}

In this work, we focus on the case where the thickness of the TI is much smaller than the wavelength of the incident

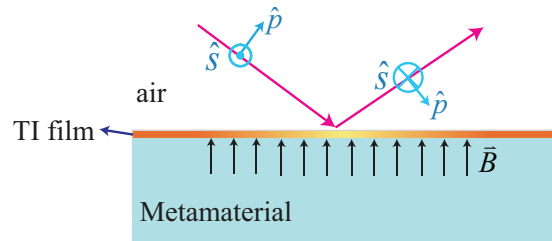

FIG. 7. (Color online) Schematic configuration for optical reflection of the TI growing on a metamaterial. Here the metamaterial means the material whose effective refraction index can be tuned in a designed way.

light but much larger than five quintuple layers (QLs), below which the interaction between the opposite surfaces of the Ti would be caused. This condition is easily achieved by growing TI films using the molecular-beam method. In this situation, following Faraday's law, ${ }^{34}$ we know that the electric field is spatially constant across the TI, so the thin TI film can be considered a surface with the surface conductivities summing the ones for both surfaces of the film. Ampere's law implies the magnetic field fulfilling the following boundary condition:

$$
\vec{H}_{u}-\vec{H}_{l}=\frac{4 \pi}{c} \vec{J}_{s} \times \hat{n},
$$

where $\vec{H}_{u}$ denotes the magnetic field in the region above the upper interface, $\vec{H}_{l}$ is for the magnetic field in the region below the bottom interface, $c$ is the velocity of light in the vacuum, $\vec{J}_{s}$ is the surface current induced by the impinging electromagnetic field, and $\hat{n}$ denotes the outward normal unit vector for the upper surface. The surface current is obtained by Ohm's law as

$$
\vec{J}_{s}=\vec{E}_{i m}\left(\sigma_{x x, l}+\sigma_{x x, u}\right)+\left(\sigma_{x y, l}+\sigma_{x y, u}\right) \hat{o} \times \vec{E}_{i m},
$$

where $\vec{E}_{i m}$ is the impinging electric field and $\hat{o}$ is the direction of the time-reversal symmetry-breaking field. By assuming the external field along the $+z$ direction and the field impinging from top to bottom (see Fig. 7, for instance), we have the reflection coefficients within the usual eigenpolarization basis consisting of the transversal electric $(s)$ polarization and the transversal magnetic $(p)$ polarization:

$$
\begin{aligned}
& r_{p, s}=\frac{4 \alpha \sigma_{x y} \sqrt{\varepsilon_{u} \mu_{u}}}{\left(\left(\frac{\varepsilon_{u} \mu_{u}}{k_{z, u}}+\frac{2 \alpha \sigma_{x x}}{\varpi \sqrt{\varepsilon_{u} \mu_{u}}}+\frac{\varepsilon_{l} \mu_{l}}{k_{z, l}}\right)\left(k_{z, u}+2 \alpha \sigma_{x x} \varpi+k_{z, l}\right)+\left(2 \alpha \sigma_{x y}\right)^{2}\right)}, \\
& r_{s, p}=\frac{4 \alpha \sigma_{x y} \sqrt{\varepsilon_{u} \mu_{u}}}{\left(\left(\frac{\varepsilon_{u} \mu_{u}}{k_{z, u}}+\frac{2 \alpha \sigma_{x x}}{\varpi \sqrt{\varepsilon_{u} \mu_{u}}}+\frac{\varepsilon_{l} \mu_{l}}{k_{z, l}}\right)\left(k_{z, u}+2 \alpha \sigma_{x x} \varpi+k_{z, l}\right)+\left(2 \alpha \sigma_{x y}\right)^{2}\right)}, \\
& r_{s, s}=\frac{\left(\left(\frac{\varepsilon_{u} \mu_{u}}{k_{z, u}}+\frac{2 \alpha \sigma_{x x}}{\varpi \sqrt{\varepsilon_{u} \mu_{u}}}+\frac{\varepsilon_{l} \mu_{l}}{k_{z, l}}\right)\left(k_{z, u}-2 \alpha \sigma_{x x} \varpi-k_{z, l}\right)+\left(2 \alpha \sigma_{x y}\right)^{2}\right)}{\left(\left(\frac{\varepsilon_{u} \mu_{u}}{k_{z, u}}+\frac{2 \alpha \sigma_{x x}}{\varpi \sqrt{\varepsilon_{u} \mu_{u}}}+\frac{\varepsilon_{l} \mu_{l}}{k_{z, l}}\right)\left(k_{z, u}+2 \alpha \sigma_{x x} \varpi+k_{z, l}\right)+\left(2 \alpha \sigma_{x y}\right)^{2}\right)}, \\
& r_{p, p}=\frac{\left(\left(-\frac{\varepsilon_{u} \mu_{u}}{k_{z, u}}+\frac{2 \alpha \sigma_{x x}}{\varpi \sqrt{\varepsilon_{u} \mu_{u}}}+\frac{\varepsilon_{l} \mu_{l}}{k_{z, l}}\right)\left(k_{z, u}+2 \alpha \sigma_{x x} \varpi+k_{z, l}\right)+\left(2 \alpha \sigma_{x y}\right)^{2}\right)}{\left(\left(\frac{\varepsilon_{u} \mu_{u}}{k_{z, u}}+\frac{2 \alpha \sigma_{x x}}{\varpi \sqrt{\varepsilon_{u} \mu_{u}}}+\frac{\varepsilon_{l} \mu_{l}}{k_{z, l}}\right)\left(k_{z, u}+2 \alpha \sigma_{x x} \varpi+k_{z, l}\right)+\left(2 \alpha \sigma_{x y}\right)^{2}\right)},
\end{aligned}
$$


where $\alpha$ is the fine-structure constant, $\sigma_{x y}$ and $\sigma_{x x}$ are the total Hall and longitudinal conductivities from both surfaces, ${ }^{34}$ $\varepsilon_{u}$ and $\mu_{u}$ are the relative permittivity and permeability for the region higher than the top surface, $\varepsilon_{l}$ and $\mu_{l}$ are the relative permittivity and permeability for the region lower than the bottom surface, $k_{z, u}$ is the $z$-component wave vector for the region higher than the top surface, $k_{z, l}$ is the $z$-component wave vector for the region lower than the bottom surface, and $\varpi=\omega / c$ is the reduced frequency. We notice that in the normal incident case (19) directly reduces to the Eqs. (27) and (28) in Ref. 34.

\section{B. Reflection spectra analysis}

Based on Eq. (19), we calculate the reflectance from the structure shown in Fig. 7 when the light shines on it normally. We assume that the TI thin film grows on a metamaterial whose refraction index can be tuned in a designed way. ${ }^{31,32}$ Here we focus on the discussion of the case when the surface gap $\Delta=0.03 \mathrm{eV}$ is larger than the chemical potential $\mu_{F}=0.01 \mathrm{eV}$. Let us first discuss the diagonal channels $\left(r_{s, s}\right.$ and $\left.r_{p, p}\right)$. By comparing (19c) and (19d), it is easy to identify
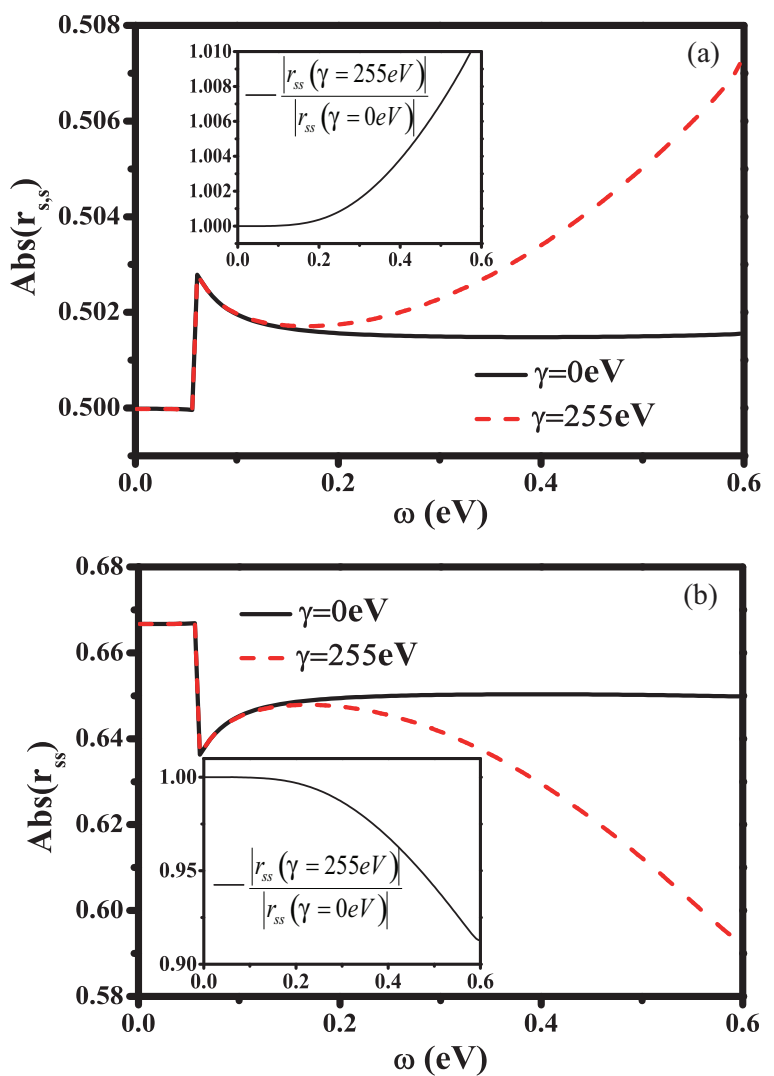

FIG. 8. (Color online) (a) Absolute value of $r_{s, s}\left[\operatorname{Abs}\left(r_{s, s}\right)\right]$ as a function of frequency. (a) The refraction index of the metamaterial is $n_{M}=3$ and is larger than that of air; (b) the refraction index of the metamaterial is $n_{M}=0.2$ and is smaller than that of air. For the insets in both (a) and (b), the curve $\frac{\mathrm{Abs}\left[r_{s, s}(\gamma=255 \mathrm{eV})\right]}{\mathrm{Abs}\left[r_{s, s}(\gamma=0 \mathrm{eV})\right]}$ is obtained by normalizing $r_{s, s}$ for the case with warping strength $\gamma=255 \mathrm{eV}$ to $r_{s, s}$ for the case with no warping. The black curve is for the case with no warping, $\gamma=0 \mathrm{eV}$, and the red dashed curve is for the case with warping strength $\gamma=255 \mathrm{eV}$. that $r_{s, s}$ and $r_{p, p}$ have little difference, so we will focus on $r_{s, s}$ here. In Fig. 8(a), we show the absolute value of $r_{s, s}$ $\left[\operatorname{Abs}\left(r_{s, s}\right)\right]$ as a function of the frequency $\omega$ for the case where the refraction index of the metamaterial $\left(n_{M}=\sqrt{\varepsilon_{l} \mu_{l}}=3\right)$ is larger than that of the vacuum $\left(n_{u}=1\right)$. From $(19 \mathrm{c})$, one can safely ignore the term on the order of $O\left(\alpha^{2}\right)$; then one can see that the real (imaginary) part of $\sigma_{x x}$ would be reflected from the real (imaginary) part of $r_{s, s}$. Under the condition $n_{M}>n_{u}$, the real part of $r_{s, s}$ would dominate. Consequently, we find that the behavior of $\operatorname{Abs}\left(r_{s, s}\right)$ is quite similar to that of the real part of $\sigma_{x x}$ (see Fig. 3), and the warping effect is shown as the increment of $\operatorname{Abs}\left(r_{s, s}\right)$ at higher frequency. For the experimental consideration, we also normalize the reflectance of the TI with warping strength $(\gamma=255 \mathrm{eV})$ to that of the TI with no warping. The normalized reflection $\left(\frac{\operatorname{Abs}\left[r_{s, s}(\gamma=255 \mathrm{eV})\right]}{\operatorname{Abs}\left[r_{s, s}(\gamma=0 \mathrm{eV})\right]}\right)$ spectrum is shown in the inset, and we find that the maximal difference due to the warping effect is only 0.01 , which is very small and quite difficult to detect.

In Fig. 8(b), we plot $\operatorname{Abs}\left(r_{s, s}\right)$ as a function of the frequency $\omega$ for the case where the refraction index of the metamaterial $\left(n_{M}=\sqrt{\varepsilon_{l} \mu_{l}}=0.2\right)$ is smaller than that of the vacuum $\left(n_{u}=\right.$ 1). In this situation, the reflectance is still dominated by its real part. Due to the sign change [the term containing $k_{z, u}-k_{z, l}$ in (19c)], the reflection spectra are simply rotated by $180^{\circ}$ in the paper plane. The normalized spectrum is also shown in the inset. This time the maximal increment due to the warping effect can be about $10 \%$ of the value without the warping term.

To see the effect of the imaginary part of $\sigma_{x x}$ in the spectra, one should set the refraction index of the metamaterial slightly smaller than that of the vacuum. For the case $n_{M}=\sqrt{\varepsilon_{l} \mu_{l}}=$ 0.9 , we plot the real part, the imaginary part, and the absolute value of $r_{s, s}$ in Fig. 9 as a function of the frequency. From the results, we find that the real part of $r_{s, s}$ is almost the same as the absolute value until the frequency reaches $0.5 \mathrm{eV}$. The effect of the imaginary part of $\sigma_{x x}$ is identified in the region highlighted in green.

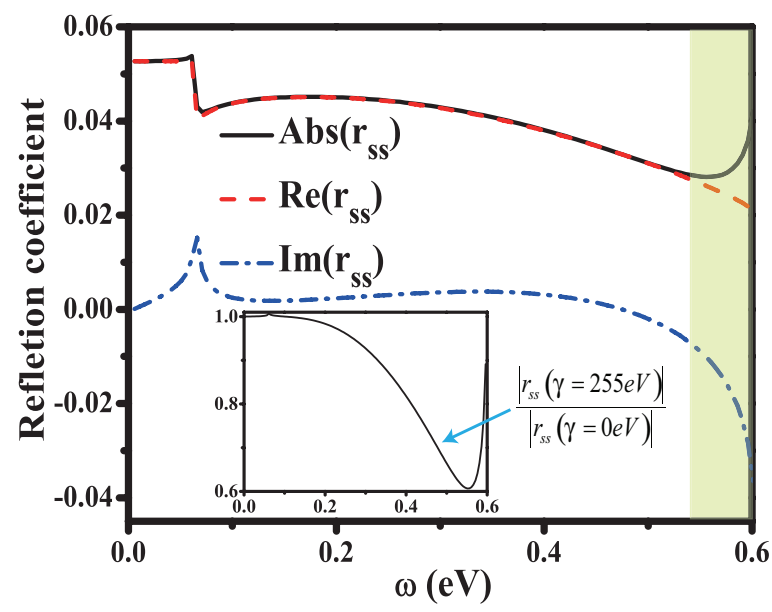

FIG. 9. (Color online) The absolute value, the real part, and the imaginary part of $r_{s, s}$ as a function of frequency when the refraction index of the metamaterial is $n_{M}=0.9$ and is slightly smaller than that of air. In the inset, the curve $\frac{\operatorname{Abs}\left[r_{s, s}(\gamma=255 \mathrm{eV})\right]}{\operatorname{Abs}\left[r_{s, s}(\gamma=0 \mathrm{eV})\right]}$ is obtained by normalizing $r_{s, s}$ for the case with warping strength $\gamma=255 \mathrm{eV}$ to $r_{s, s}$ for the case with no warping. The green highlighted region illustrates that the imaginary part of $\sigma_{x x}$ begins to play a role. 


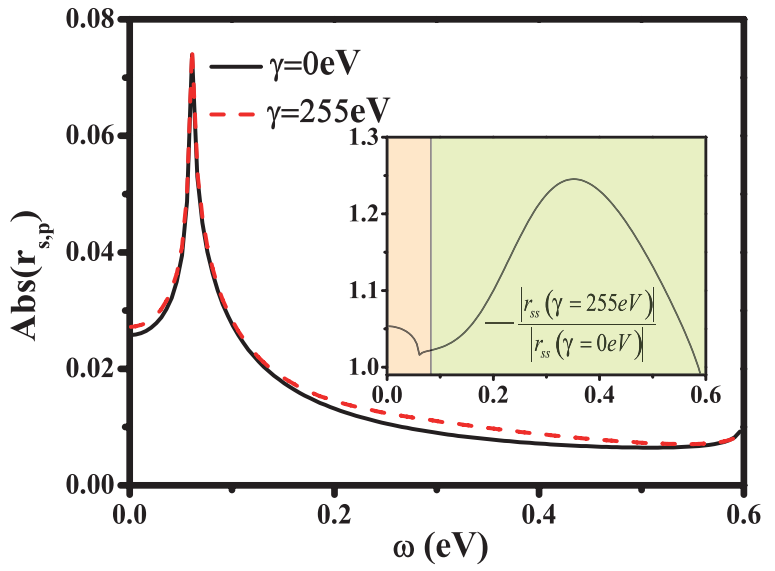

FIG. 10. (Color online) The absolute value $r_{s, p}$ as a function of frequency when the refraction index of the metamaterial is $n_{M}=0.01$. The black curve is for the $\gamma=0 \mathrm{eV}$ case, and the red dashed curve is for the $\gamma=255 \mathrm{eV}$ case. In the inset, the curve $\frac{\operatorname{Abs}\left[r_{s, p}(\gamma=255 \mathrm{eV})\right]}{\mathrm{Abs}\left[r_{s, p}(\gamma=0 \mathrm{eV})\right]}$ is obtained by normalizing $r_{s, p}$ for the case with warping strength $\gamma=255 \mathrm{eV}$ to $r_{s, p}$ for the case with no warping. In the inset, the region dominated by the real part of $\sigma_{x y}$ is highlighted in orange, and the region dominated by the imaginary part of $\sigma_{x y}$ is highlighted in green.

Next, we consider the off-diagonal reflection channels, for which $r_{s, p}=r_{p, s}$ from Eq. (19). Generally, the absolute value of $r_{s, p}$ is small, but as the refraction index of the metamaterial approaches zero, the reflection signal may be large enough to detect. In Fig. 10, the absolute value of $r_{s, p}$ is shown as a function of the frequency $\omega$ for the case where the refraction index of the metamaterial $n_{M}=0.01$. We find that the behavior of $\operatorname{Abs}\left(r_{s, p}\right)$ is dominated by the real part of $r_{s, p}$ at low frequency and by the imaginary part of $r_{s, p}$ at high frequency. Therefore, at low frequency the behavior of $\operatorname{Abs}\left(r_{s, p}\right)$ is similar to that of the real part of $\sigma_{x y}$, and similar behavior to that of the imaginary part of $\sigma_{x y}$ is shown at higher frequency. We also normalize the reflectance of the TI with warping strength $\gamma=255 \mathrm{eV}$ to that of the TI with no warping. The normalized reflection $\left(\frac{\mathrm{Abs}\left[r_{s, p}(\gamma=255 \mathrm{eV})\right]}{\mathrm{Abs}\left[r_{s, p}(\gamma=0 \mathrm{eV})\right]}\right)$ spectrum is shown in the inset. In the inset, the region dominated by the real part of $\sigma_{x y}$ is highlighted in orange, and the region dominated by the imaginary part of $\sigma_{x y}$ is shown in green. At high frequency, obvious differences induced by the warping can be determined.

The warping-induced difference at low frequency shown in the inset of Fig. 10 has a close relation with the choice of the energy cutoff. With the increasing of the energy cutoff, both $\sigma_{x y}$ for the cases with and without warping saturate to the half integer, which means that the difference in the spectra will diminish accordingly. The lower the energy cutoff is, the more obvious the difference is.

\section{Reflection coefficients under the conditions $\Delta>\mu$ and $\Delta \gg \omega$}

As noted, when the energy cutoff is finite, the optical Hall conductivity is not perfectly quantized to the half integer (see Fig. 6). Moreover, the value of the conductivity will increase with the warping strength [see Fig. 6(b)]. Below, we explore the possible optical signatures of the warping effects at low frequency due to the finite-energy cutoff. Under the conditions $\Delta>\mu$ and $\Delta \gg \omega$, only the optical Hall conductivity (real part) is nonvanishing. Therefore, one can redefine the new eigenpolarizations $\hat{e}_{1}$ and $\hat{e}_{2}$ to substitute for the $s$ and $p$ polarizations, so that the incident field can be related to the reflected field through

$$
E_{1}^{r} \hat{e}_{1}=r_{1,1} E_{1}^{i} \hat{e}_{1}, \quad E_{2}^{r} \hat{e}_{1}=r_{2,2} E_{2}^{i} \hat{e}_{2},
$$

which means that there is no cross correlation between the two eigenpolarizations. We find that the new eigenpolarizations relate to the original $s$ and $p$ polarizations as

$$
\left(\begin{array}{l}
\hat{e}_{1} \\
\hat{e}_{2}
\end{array}\right)=\left(\begin{array}{cc}
\cos \phi & \sin \phi \\
-\sin \phi & \cos \phi
\end{array}\right)\left(\begin{array}{l}
\hat{s} \\
\hat{p}
\end{array}\right),
$$

where the rotation angle is given by

$$
\tan (2 \phi)=\frac{4 \alpha \sigma_{x y} \sqrt{\varepsilon_{u} \mu_{u}}}{\varepsilon_{u} \mu_{u}-\varepsilon_{l} \mu_{l}-\left(2 \alpha \sigma_{x y}\right)^{2}} .
$$

Then the reflection coefficient can be written in a way similar to the Fresnel equations as

$$
r_{1,1}=\frac{\bar{n} \cos \varphi-\bar{n}^{\prime} \cos \varphi^{\prime}}{\bar{n} \cos \varphi+\bar{n}^{\prime} \cos \varphi^{\prime}}, \quad r_{2,2}=\frac{\overline{n^{\prime}} \cos \varphi-\bar{n} \cos \varphi^{\prime}}{\overline{n^{\prime} \cos \varphi+\bar{n} \cos \varphi^{\prime}}},
$$

where $\varphi$ and $\varphi^{\prime}$ are the incident angle and refraction angle, respectively, and $\bar{n}$ and $\bar{n}^{\prime}$ are the effective fraction indexes of the form

$$
\begin{aligned}
& \bar{n}=\frac{1}{2}\left[\begin{array}{c}
\sqrt{\left(\sqrt{\varepsilon_{u} \mu_{u}}+\sqrt{\varepsilon_{l} \mu_{l}}\right)^{2}+\left(2 \alpha \sigma_{x y}\right)^{2}} \\
\pm \sqrt{\left(\sqrt{\varepsilon_{u} \mu_{u}}-\sqrt{\varepsilon_{l} \mu_{l}}\right)^{2}+\left(2 \alpha \sigma_{x y}\right)^{2}}
\end{array}\right], \\
& \overline{n^{\prime}}=\frac{1}{2}\left[\begin{array}{c}
\sqrt{\left(\sqrt{\varepsilon_{u} \mu_{u}}+\sqrt{\varepsilon_{l} \mu_{l}}\right)^{2}+\left(2 \alpha \sigma_{x y}\right)^{2}} \\
\mp \sqrt{\left(\sqrt{\varepsilon_{u} \mu_{u}}-\sqrt{\varepsilon_{l} \mu_{l}}\right)^{2}+\left(2 \alpha \sigma_{x y}\right)^{2}}
\end{array}\right],
\end{aligned}
$$

where plus (+) in the first equation and minus ( -$)$ in the second equation are chosen when $\varepsilon_{u} \mu_{u} \geqslant \varepsilon_{l} \mu_{l}+\left(2 \alpha \sigma_{x y}\right)^{2}$. We notice that this expression can be reduced to the $\theta$ term $^{35}$ descriptions in the language of axion electrodynamics. ${ }^{36}$ For our current situation with $\Delta>\mu$ and $\Delta \gg \omega$, only the real part of the optical Hall conductivity is nonvanishing and quantized. After matching the boundary conditions, the magnetic fields on the two sides of a TI surface are linked by the surface Hall current, which is proportional to the electric field (Ohm's law). Because the Hall conductivity is quantized, a quantized magnetoelectric coupling is realized. The coupling processes described above can be conveniently described by a Lagrangian density $L_{\theta}=$ $\frac{\alpha \theta}{4 \pi^{2}} \vec{E} \cdot \vec{B}$ with $\theta=\pi$ for the current case (more generally, $\theta$ can be an odd integer multiple of $\pi$ ). The Lagrangian density is called the $\theta$ term in the literature of axion electrodynamics.

In the following, we will consider the Goos-Hänchen effect and the Brewster angle measurement to detect the difference induced by the warping term by assuming a finite-energy cutoff for the surface states.

\section{Goos-Hänchen effect}

When the refraction index of the metamaterial is lower than that of air and the incident angle is larger than the critical angle $\varphi_{c}=\sin ^{-1}\left(\frac{\sqrt{\varepsilon_{l} \mu_{l}}}{\sqrt{\varepsilon_{u} \mu_{u}}}\right)$, total reflection occurs. In this situation, the Fresnel-like reflection coefficients become complex and have 

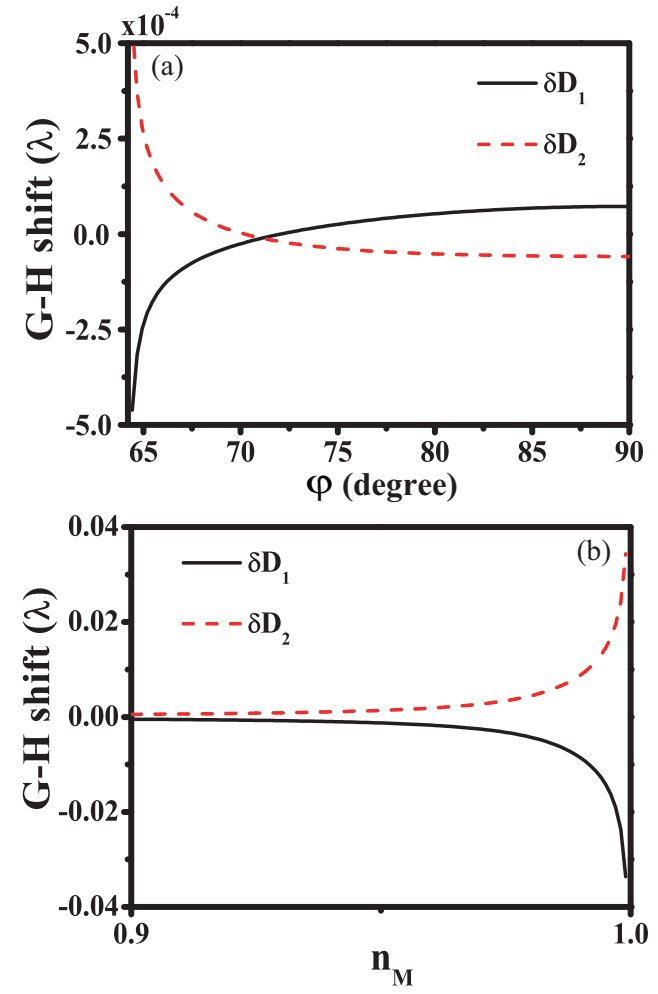

FIG. 11. (Color online) (a) $\delta D_{i}$ (defined in the text) as a function of the incident angle, where we set the refraction index of the metamaterial as $n_{M}=\sqrt{\varepsilon_{l} \mu_{l}}=0.9$. (b) The maximal G-H shift $\delta D_{i}$ as a function of the refraction index $n_{M}$ of the metamaterial.

the form $r_{i, i}=\exp \left(i \vartheta_{i}\right)$, with $i=1,2$. The Goos-Hänchen (G-H) shift in the reflected beam is defined as $D_{i}=$ $-\frac{\lambda}{2 \pi} \frac{d \vartheta_{i}}{d \varphi},{ }^{35,37}$ where $\lambda$ is the wavelength in air, $\vartheta_{i}$ is the phase angle of $r_{i, i}$, and $\varphi$ is the incident angle.

To consider the warping effect on the G-H shifts, we consider the TI film $\left(\mathrm{Bi}_{2} \mathrm{Te}_{3}\right)$ with warping strength $\gamma=$ $255 \mathrm{eV}$, and we compare the results with those of the TI film with no warping. To make a fair comparison, we assume that both of them have the same energy cutoff $\varepsilon_{c}=0.3 \mathrm{eV}$, the same chemical potential $\mu_{F}=0.01 \mathrm{eV}$, and the same surface gap $\Delta=0.03 \mathrm{eV}$. The differences in the G-H shifts for the two cases are defined as $\delta D_{i}=D_{i}^{B i_{2} T e_{3}}-D_{i}^{\text {no-warping }}$.

In Fig. 11(a), we first plot $\delta D_{i}$ as a function of the incident angle $\varphi$ for the incident frequency $\omega=0.001 \mathrm{eV}$, where we set the refraction index of the metamaterial as $n_{M}=\sqrt{\varepsilon_{l} \mu_{l}}=$ 0.9 and the refraction index of the air as $n_{A}=\sqrt{\varepsilon_{u} \mu_{u}}=1$. From Fig. 11(a), we find that the maximal G-H shift (absolute value) occurs when the incident angle is the critical angle. In Fig. 11(b), we plot the maximal G-H shift as a function of the refraction index of the metamaterial. We notice that when the refraction index of the metamaterial approaches that of the air, the G-H shift can be on the order of $0.01 \lambda$, which can be measured by means of the interference method. ${ }^{38}$

\section{E. Brewster angle}

Because Eq. (23) is a close analog to the Fresnel equations, one can expect that when the incident wave is linearly polarized along one of the eigenpolarizations and incident at the Brewster angle, the reflected field can be vanishing. By comparing

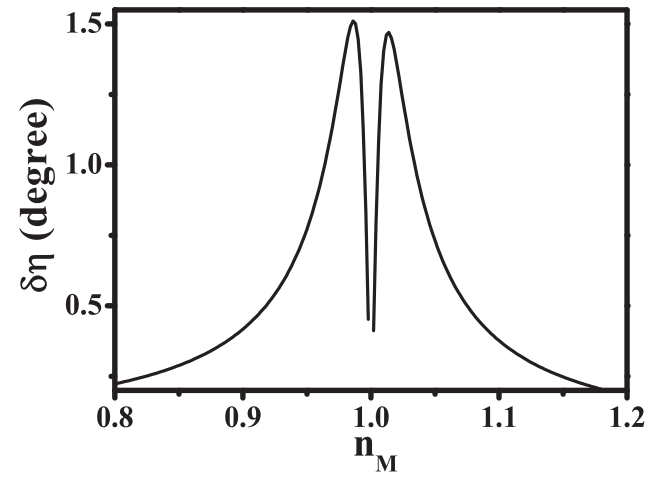

FIG. 12. $\delta \eta$ (defined in the text) as a function of the refraction index $n_{M}$ of the metamaterial. When the refraction index of the metamaterial is $n_{M}=0.986$ or $n_{M}=1.014$, the angle shift induced by warping can be as large as $1.5^{\circ}$, which is experimentally accessible.

Eq. (23) with the Fresnel equations, we identify that $r_{2,2}$ can be zero when the incident angle fulfills

$$
\tan \varphi_{B}=\sqrt{\frac{\varepsilon_{l} \mu_{l}}{\varepsilon_{u} \mu_{u}}} \sqrt{\left|\frac{1-\left(\overline{n^{\prime}} / \bar{n}\right)^{2}}{1-\frac{\varepsilon_{l} \mu_{l}}{\varepsilon_{u} \mu_{u}}}\right|},
$$

where $\bar{n}^{\prime}$ and $\bar{n}$ are defined in Eq. (24). For the real detection, one can let the incident field impinge on the structure shown in Fig. 7 with the Brewster angle. By rotating the polarization, one can find the direction of $\hat{e}_{2}$. We denote the angle between $\hat{e}_{2}$ and $\hat{p}$ as the angle shift $\eta$. The angle shift of $\eta$ can be calculated for $\mathrm{Bi}_{2} \mathrm{Te}_{3}$ and the TI with no warping. The difference in the angle shift $\eta$ induced by the warping effect is defined as $\delta \eta=\eta^{\mathrm{Bi}_{2} \mathrm{Te}_{3}}-\eta^{\text {no-warping }}$. In Fig. 12, we plot $\delta \eta$ as a function of the refraction index of the metamaterial $n_{M}$. We notice that the angle shift difference induced by the warping effect can reach $1.5^{\circ}$ when the dielectric constant of the metamaterial is around $n_{M}=0.986$ or $n_{M}=1.014$.

\section{F. Discussion of the case with $\Delta<\mu_{F}$}

In the above sections, we focused on the presentation of the possible optical signatures when the chemical potential $\mu_{F}$ is smaller than the surface gap $\Delta$. Here we would like to discuss the possible features for the case when the chemical potential $\mu_{F}$ is larger than the surface gap $\Delta$. For this case, it is easier to identify the warping effect from the low-frequency reflection spectra, from which the effect of the warping term on $\operatorname{Im}\left[\sigma_{x x}^{\text {intra }}(\omega)\right]$ can be observed. To demonstrate it, we assume that the chemical potentials of the TIs under consideration are large (i.e., $\mu_{F}>0.15 \mathrm{eV}$ ). In this situation, the dissipative conductivities contributed by the interband processes $\left(\operatorname{Re}\left[\sigma_{x x}^{\text {inter }}(\omega)\right]\right.$ and $\left.\operatorname{Im}\left[\sigma_{x y}^{\text {inter }}(\omega)\right]\right)$ simply vanish at low frequency [see Eqs. (13) and (15) or see Figs. 3 and 4]. In the meanwhile, the dissipationless conductivities from the interband processes $\left(\operatorname{Im}\left[\sigma_{x x}^{\text {inter }}(\omega)\right]\right.$ and $\left.\operatorname{Re}\left[\sigma_{x y}^{\text {inter }}(\omega)\right]\right)$ are much smaller than $\operatorname{Im}\left[\sigma_{x x}^{\text {intra }}(\omega)\right]$ in magnitude when the frequency is low (see Figs. 2, 5, and 6). On the other hand, the value of $\operatorname{Im}\left[\sigma_{x x}^{\text {intra }}(\omega)\right]$ would change obviously with the variation of the warping strength $\gamma$ [see Fig. 2(b), for example]. Therefore, by using a structure similar to that shown in Fig. 7 in experiments, the change in $\operatorname{Im}\left[\sigma_{x x}^{\text {intra }}(\omega)\right]$ as a result of the warping effect can be detected in the low-frequency reflection spectra. 


\section{CONCLUSION}

In this work, we have studied the influences of the warping term on the optical response of TIs. By using Kubo's formula, we systematically analyzed how the warping effect and the nonzero surface gap affect the optical conductivities. To discuss the possible signatures of the warping effect in optics, we considered a special structure: the TI thin film growing on a metamaterial. We found that by properly tuning the refraction index of the metamaterial, all the features of the optical conductivities due to the warp term can be detected and identified optically in the spectra. For real samples, the finite-energy cutoff of the surface states may cause imperfect quantization of the optical Hall conductivity at low frequency. Under these conditions, the warping term would have some effects on the imperfectly quantized Hall conductivity. The possibility of using the Goos-Hänchen effect and Brewster angle measurement to detect the warping effect on the imperfectly quantized optical Hall conductivity was suggested.

\section{ACKNOWLEDGMENTS}

We would like to thank Professor K. T. Law for useful comments and suggestions. This work is supported by Research Grant No. HKUST2/CRF/11G.

\section{APPENDIX: THE EFFECT OF PARTICLE-HOLE ASYMMETRY}

The particle-hole asymmetry in TI materials can be modeled by an additional quadratic background term, so the Hamiltonian becomes ${ }^{11}$

$$
\hat{\mathcal{H}}=\frac{k^{2}}{2 m^{*}}+v_{F}\left(k_{x} \hat{\sigma}_{y}-k_{y} \hat{\sigma}_{x}\right)+\frac{\gamma}{2}\left(k_{+}^{3}+k_{-}^{3}\right) \hat{\sigma}_{z}+\Delta \sigma_{z},
$$

where $m^{*}$ is the effective mass of the quasiparticle and its value is typically small. ${ }^{10,16}$ The particle-hole asymmetry is tuned by the factor $m^{*}$ : when $\frac{1}{m^{*}}=0$, the particle-hole symmetry is recovered. The additional background term would introduce a few differences. First of all, both the velocity operators $\hat{v}_{x}$ and $\hat{v}_{y}$ include an additional term proportional to $1 / m^{*}$. Due to the orthogonality between the conducting band and the valance band, these terms only change the intraband expectations of the velocity operators and leave the interband expectations unchanged. Second, the additional term also causes the differences when one transforms the integral according to the momentum $k$ into the one according to the energy of the conducting band $\varepsilon_{+}$, for example,

$$
\int k d k=\int d \varepsilon_{+} \frac{1}{D(k, \theta)}
$$

with $D(k, \theta)=\frac{1}{m^{*}}+\frac{6 \gamma\left(\gamma k^{3} \cos 3 \theta+\Delta\right) k \cos 3 \theta+2 v_{F}^{2}}{\varepsilon_{+}-\varepsilon_{-}}$and $\varepsilon_{ \pm}(k, \theta)=$ $\frac{k^{2}}{2 m^{*}} \pm \sqrt{\left(\gamma k^{3} \cos 3 \theta+\Delta\right)^{2}+\left(v_{F} k\right)^{2}}$.

Then, following the procedures shown in the text, one can find that the intraband Hall conductivity is simply zero [due to the symmetry of $\left(v_{x}\right)_{+,+}\left(v_{y}\right)_{+,+}$], and the intraband longitudinal conductivity is given by

$$
\sigma_{x x}^{\text {intra }}(\omega)=\frac{i}{2 \pi \omega} \Theta\left(\mu_{F}-\Delta\right) \int \frac{d \theta \mu_{F}\left|\left(v_{x}\right)_{+,+}\right|^{2}}{D(k, \theta)},
$$

where the wave vector $k$ in the above equation is determined by

$$
\left(\mu_{F}-\frac{k^{2}}{2 m^{*}}\right)^{2}=\left(\gamma k^{3} \cos 3 \theta+\Delta\right)^{2}+\left(v_{F} k\right)^{2},
$$

and the expectation value $\left|\left(v_{x}\right)_{+,+}\right|^{2}$ is given by

$$
\begin{aligned}
& \left|\left(v_{x}\right)_{+,+}\right|^{2} \\
& \quad=\left(\frac{k \cos \theta}{m^{*}}+3 \gamma k^{2} \cos 2 \theta \cos \beta+v_{F} \sin \beta \cos \theta\right)^{2},
\end{aligned}
$$

with $\cos \beta=\frac{2\left(k^{3} \cos 3 \theta_{\vec{k}}+\Delta\right)}{\varepsilon_{+}-\varepsilon_{-}}$.

For the interband contribution to the optical longitudinal conductivities, we can obtain them by following Kubo's formula (10) by substituting $2 \varepsilon_{+}$with $\varepsilon_{+}-\varepsilon_{-}$. Because of the function $g\left(\varepsilon_{+}\right)=\Theta\left(\varepsilon_{+}-\mu_{F}\right)$, the integral can be conducted within the conducting band once one assumes the Fermi surface crossing the conducting band. Then the real part and the imaginary part of the conductivity are given by

$$
\begin{aligned}
\operatorname{Re} & {\left[\sigma_{x x}^{\text {inter }}(\omega)\right] } \\
= & \frac{1}{2} \int \frac{d \theta\left|\left(v_{x}\right)_{+,-}\right|^{2} \Theta\left(\varepsilon_{+}-\max \left(\Delta, \mu_{F}\right)\right)}{D(k, \theta)} \frac{\varepsilon_{+}}{\left(\varepsilon_{+}-\varepsilon_{-}\right)^{2}}, \\
\operatorname{Im}[ & \left.\sigma_{x x}^{\text {inter }}(\omega)\right] \\
= & \frac{1}{2 \pi} \iint \frac{d \theta d \varepsilon_{+}\left|\left(v_{x}\right)_{+,-}\right|^{2}}{D(k, \theta)} \frac{\Theta\left(\varepsilon_{+}-\mu_{F}\right)}{\varepsilon_{+}-\varepsilon_{-}} \\
& \times\left[\frac{1}{\omega+2\left(\varepsilon_{+}-\varepsilon_{-}\right)}+\frac{\omega-2\left(\varepsilon_{+}-\varepsilon_{-}\right)}{\left[\omega-\left(\varepsilon_{+}-\varepsilon_{-}\right)\right]^{2}+4 / \tau^{2}}\right] .
\end{aligned}
$$

In Eq. (A6a), we have already integrated according to the energy. Because of the delta function $\delta\left(\omega-\varepsilon_{+}+\varepsilon_{-}\right)$, a factor $\frac{\varepsilon_{+}}{\varepsilon_{+}-\varepsilon_{-}}$appears in the equation. One can easily find that when $\frac{1}{m^{*}} \rightarrow 0$, Eqs. (A6) reduce to Eq. (11). The wave vector $k$ and the energy $\varepsilon_{+}$in (A6a) are determined by $\left(\gamma k^{3} \cos 3 \theta_{\vec{k}}+\right.$ $\Delta)^{2}+\left(v_{F} k\right)^{2}=\omega^{2} / 4$, and the wave vector $k$ in $(\mathrm{A} 6 \mathrm{~b})$ is determined by $\left(\varepsilon_{+}-\frac{k^{2}}{2 m^{*}}\right)^{2}=\left(\gamma k^{3} \cos 3 \theta+\Delta\right)^{2}+\left(v_{F} k\right)^{2}$.

Similarly, the interband contribution to the optical Hall conductivity can be evaluated as

$$
\begin{aligned}
\operatorname{Re} & {\left[\sigma_{x y}^{\text {inter }}(\omega)\right] } \\
= & \frac{1}{2 \pi} \iint \frac{d \theta d \varepsilon \operatorname{Im}\left[\left(v_{x}\right)_{+,-}\left(v_{y}\right)_{-,+}\right]}{D(k, \theta)} \frac{\Theta\left(\varepsilon_{+}-\mu_{F}\right)}{\varepsilon_{+}-\varepsilon_{-}} \\
& \times\left[\frac{1}{\omega+2\left(\varepsilon_{+}-\varepsilon_{-}\right)}-\frac{\omega-2\left(\varepsilon_{+}-\varepsilon_{-}\right)}{\left[\omega-\left(\varepsilon_{+}-\varepsilon_{-}\right)\right]^{2}+4 / \tau^{2}}\right],
\end{aligned}
$$

$$
\begin{aligned}
\operatorname{Im} & {\left[\sigma_{x y}^{\text {inter }}(\omega)\right] } \\
= & \frac{1}{2} \int d \theta \operatorname{Im}\left[\left(v_{x}\right)_{+,-}\left(v_{y}\right)_{-,+}\right] \\
& \times \frac{\Theta\left(\varepsilon_{+}-\max \left(\Delta, \mu_{F}\right)\right)}{D(k, \theta)} \frac{\varepsilon_{+}}{\left(\varepsilon_{+}-\varepsilon_{-}\right)^{2}} .
\end{aligned}
$$




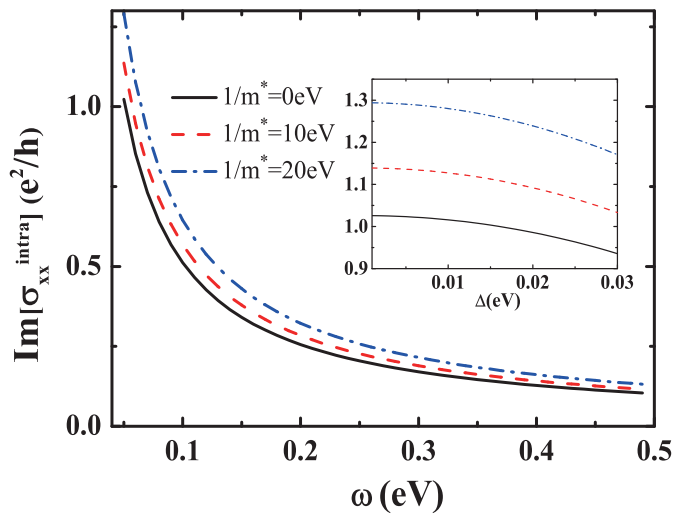

FIG. 13. (Color online) $\operatorname{Im}\left[\sigma_{x x}^{\text {intra }}(\omega)\right]$ as a function of frequency $\omega$ for three different effective masses, where the chemical potential is $\mu_{F}=0.1 \mathrm{eV}$, the surface gap is $\Delta=0.005 \mathrm{eV}$, and the warping strength is $\gamma=255 \mathrm{eV}$. In the inset, $\operatorname{Im}\left[\sigma_{x x}^{\text {intra }}(\omega)\right]$ is plotted as a function of surface gap $\Delta$, where the chemical potential is $\mu_{F}=$ $0.1 \mathrm{eV}$, the frequency is $\omega=0.05 \mathrm{eV}$, and the warping strength is $\gamma=255 \mathrm{eV}$.

One can find that the above results can be reduced to Eq. (15) when the particle-hole symmetry is recovered (i.e., $\left.1 / m^{*}=0\right)$.
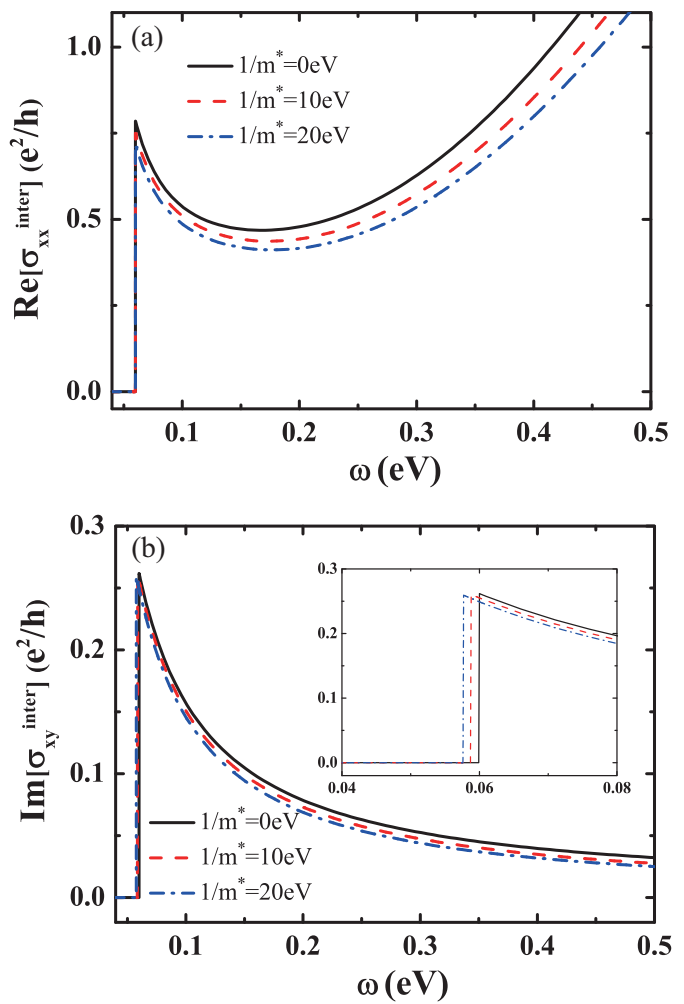

FIG. 14. (Color online) (a) $\operatorname{Re}\left[\sigma_{x x}^{\text {inter }}(\omega)\right]$ as a function of frequency $\omega$ for three different effective masses, where the chemical potential $\left(\mu_{F}=0.01 \mathrm{eV}\right)$ is set to be smaller than the surface gap $(\Delta=0.03 \mathrm{eV})$ and the warping strength is $\gamma=255 \mathrm{eV}$. (b) $\operatorname{Im}\left[\sigma_{x y}^{\text {inter }}(\omega)\right]$ as a function of frequency $\omega$ for three effective masses, where the chemical potential $\left(\mu_{F}=0.03 \mathrm{eV}\right)$ is set to be larger than the surface gap $(\Delta=0.01 \mathrm{eV})$ and the warping strength is $\gamma=255 \mathrm{eV}$. In the inset a close-up around the onset frequency $0.06 \mathrm{eV}$ is shown.
In Fig. 13 we plot the imaginary part of the optical longitudinal conductivity contributed by the intraband processes as a function of frequency $\omega$ for three different effective masses. In the inset of Fig. 13, we show the surface gap dependence of $\operatorname{Im}\left[\sigma_{x x}^{\text {intra }}(\omega)\right]$ for the same three effective masses. It can be seen that neither the frequency-dependent nor the surfacegap-dependent behavior of $\operatorname{Im}\left[\sigma_{x x}^{\text {intra }}(\omega)\right]$ is changed by the particle-hole asymmetry. Moreover, the larger the value of $1 / m^{*}$ is, the larger $\operatorname{Im}\left[\sigma_{x x}^{\text {intra }}(\omega)\right]$ is.

Next, we turn to the discussion of the interband contributions. From Eqs. (A6a) and (A7b), one can see that the expression of $\operatorname{Re}\left[\sigma_{x x}^{\text {inter }}(\omega)\right]$ is similar to that $\operatorname{Im}\left[\sigma_{x y}^{\text {inter }}(\omega)\right]$, which means that the influence of the particle-hole asymmetry on the two conductivities should also be similar. In Fig. 14(a), $\operatorname{Re}\left[\sigma_{x x}^{\mathrm{inter}}(\omega)\right]$ is plotted as a function of frequency $\omega$ for three different effective masses, where in the calculation the chemical potential is set to be smaller than the surface gap. It can be observed that the value of $\operatorname{Re}\left[\sigma_{x x}^{\text {inter }}(\omega)\right]$ decreases with the increment of $1 / \mathrm{m}^{*}$, although the trends of the variations of $\operatorname{Re}\left[\sigma_{x x}^{\text {inter }}(\omega)\right]$ according to the frequency $\omega$ are similar for the three different effective masses. For a contrast, in Fig. 14(b), we plot $\operatorname{Im}\left[\sigma_{x y}^{\text {inter }}(\omega)\right]$ as a function of frequency $\omega$ for the same three effective masses. Here we assume that the chemical potential is larger than the surface gap. Similarly, we also find that the larger $1 / m^{*}$ is, the smaller the value of $\operatorname{Im}\left[\sigma_{x y}^{\text {inter }}(\omega)\right]$
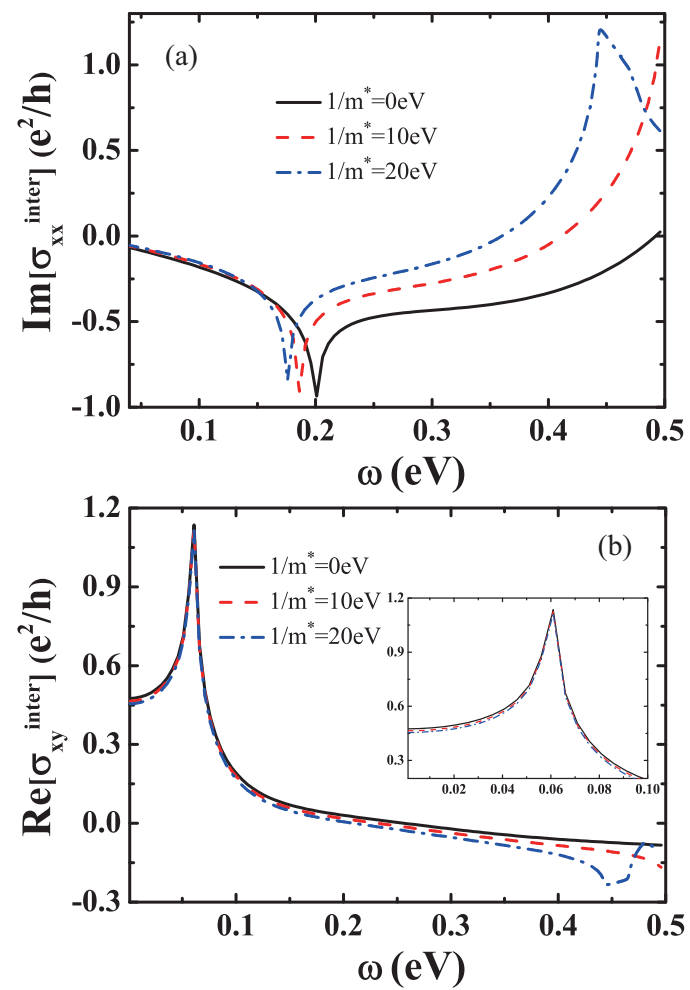

FIG. 15. (Color online) (a) $\operatorname{Im}\left[\sigma_{x x}^{\text {inter }}(\omega)\right]$ as a function of frequency $\omega$ for three different effective masses, where the chemical potential $\left(\mu_{F}=0.1 \mathrm{eV}\right)$ is set to be larger than the surface gap $(\Delta=$ $0.01 \mathrm{eV}$ ) and the warping strength is $\gamma=255 \mathrm{eV}$. (b) $\operatorname{Re}\left[\sigma_{x y}^{\text {inter }}(\omega)\right]$ as a function of frequency $\omega$ for three effective masses, where the chemical potential $\left(\mu_{F}=0.01 \mathrm{eV}\right)$ is set to be smaller than the surface gap $(\Delta=0.03 \mathrm{eV})$ and the warping strength is $\gamma=255 \mathrm{eV}$. In the inset a close-up of $\operatorname{Re}\left[\sigma_{x y}^{\text {inter }}(\omega)\right]$ at low frequency is shown. 
is. However, when the chemical potential is larger than the surface gap, the onset frequencies are different for different effective masses [see the inset of Fig. 14(b)]. To understand this feature, we recall three points: (1) from Eqs. (A6a) and (A7b), the energy relates to the frequency as $\varepsilon_{+}=\omega / 2+\frac{k^{2}}{2 m^{*}}$, which means that the larger $1 / m^{*}$ is, the larger the deviation of $\varepsilon_{+}$is from $\omega / 2$. (2) At $\varepsilon_{+}=\Delta$, the wave vector is $k=0$, which means $\varepsilon_{+}=\omega$. (3) In Eqs. (A6a) and (A7b) there is a step function $\Theta\left(\varepsilon_{+}-\max \left(\Delta, \mu_{F}\right)\right)$. Therefore, when the chemical potential is larger than the surface gap, the larger $1 / m^{*}$ leads to the condition $\varepsilon_{+}>\max \left(\Delta, \mu_{F}\right)$, which causes the step function to equal 1 , realized with a smaller $\omega$, which is exactly the results shown in Fig. 14(b).

Further, in Fig. 15(a) we plot $\operatorname{Im}\left[\sigma_{x x}^{\text {inter }}(\omega)\right]$ as a function of frequency $\omega$ for three different effective masses, where we assume that the chemical potential is larger than the surface gap. The dips in the curves correspond to the position $\omega-2\left(\varepsilon_{+}-\varepsilon_{-}\right)$. The shift of the position of the dip is due to the same reason as the shift of onset frequency in Fig. 14(b). From the results shown in Fig. 15(a), we notice that in the highfrequency regime $(\omega>0.4 \mathrm{eV})$, the frequency-dependent behavior of $\operatorname{Im}\left[\sigma_{x x}^{\text {inter }}(\omega)\right]$ is changed remarkably by the particle-hole asymmetry: when the particle-hole asymmetry is large enough $\left(1 / m^{*}=20 \mathrm{eV}\right.$; the blue dash-dotted curve), a peak in the conductivity is observed at around $\omega \sim 0.45 \mathrm{eV}$. We have to note that the frequency is much lower than the cutoff frequency of the surface model $\left(\omega_{c}=2 \varepsilon_{c}=0.6 \mathrm{eV}\right)$; the observed peak in the conductivity is not an artifact induced by the energy cutoff of the surface model. In Fig. 15(b), we plot $\operatorname{Re}\left[\sigma_{x y}^{\text {inter }}(\omega)\right]$ as a function of frequency $\omega$. Because the surface gap $\Delta$ is larger than the chemical potential $\mu_{F}$, the peak in the conductivity at $\omega=2 \Delta=0.6 \mathrm{eV}$ is not shifted by the particle-hole asymmetry. In the high-frequency regime, the remarkable difference induced by the particle-hole asymmetry can be seen (i.e., the dip at around $\omega=0.45 \mathrm{eV}$ in the blue dash-dotted curve). *phwen@ust.hk

${ }^{1}$ M. Z. Hasan and C. L. Kane, Rev. Mod. Phys. 82, 3045 (2010).

${ }^{2}$ X. L. Qi and S. C. Zhang, Rev. Mod. Phys. 83, 1057 (2011).

${ }^{3}$ J. E. Moore, Nature (London) 464, 194 (2010).

${ }^{4}$ C. L. Kane and E. J. Mele, Phys. Rev. Lett. 95, 146802 (2005); 95, 226801 (2005).

${ }^{5}$ B. A. Bernevig, T. L. Hughes, and S.-C. Zhang, Science 314, 1757 (2006).

${ }^{6}$ L. Fu, C. L. Kane, and E. J. Mele, Phys. Rev. Lett. 98, 106803 (2007).

${ }^{7}$ X.-L. Qi, T. L. Hughes, and S.-C. Zhang, Phys. Rev. B 78, 195424 (2008).

${ }^{8}$ D. Hsieh, D. Qian, L. Wray, Y. Xia, Y. S. Hor, R. J. Cava, and M. Z. Hasan, Nature (London) 452, 970 (2008).

${ }^{9}$ H. Zhang, C. X. Liu, X. L. Qi, X. Dai, Z. Fang, and S. C. Zhang, Nat. Phys. 5, 438 (2009).

${ }^{10}$ H. Z. Lu, W. Y. Shan, W. Yao, Q. Niu, and S. Q. Shen, Phys. Rev. B 81, 115407 (2010).

${ }^{11}$ L. Fu, Phys. Rev. Lett. 103, 266801 (2009).

${ }^{12}$ X.-L. Qi, R. Li, J. Zang, and S.-C. Zhang, Science 323, 1184 (2009).

${ }^{13}$ W. K. Tse and A. H. MacDonald, Phys. Rev. Lett. 105, 057401 (2010).

${ }^{14}$ A. G. Grushin and A. Cortijo, Phys. Rev. Lett. 106, 020403 (2011).

${ }^{15}$ X. Xiao, S. Li, K. T. Law, B. Hou, C. T. Chan, and W. Wen, Phys. Rev. B 87, 205424 (2013).

${ }^{16}$ Y. L. Chen, J. G. Analytis, J. H. Chu, Z. K. Liu, S. K. Mo, X. L. Qi, H. Zhang, D. Lu, X. Dai, Z. Fang, S. C. Zhang, I. R. Fisher, Z. Hussain, and Z. X. Shen, Science 325, 178 (2009).

${ }^{17}$ C. X. Liu, X. L. Qi, H. J. Zhang, X. Dai, Z. Fang, and S. C. Zhang, Phys. Rev. B 82, 045122 (2010).

${ }^{18}$ K. Nomura and N. Nagaosa, Phys. Rev. Lett. 106, 166802 (2011).
${ }^{19}$ T. Yokoyama and S. Murakami, Phys. Rev. B 83, 161407 (2011).

${ }^{20}$ R. Takahashi and S. Murakami, Phys. Rev. Lett. 107, 166805 (2011).

${ }^{21}$ P. M. Ostrovsky, I. V. Gornyi, and A. D. Mirlin, Phys. Rev. Lett. 105, 036803 (2010).

${ }^{22}$ A. A. Taskin, S. Sasaki, K. Segawa, and Y. Ando, Phys. Rev. Lett. 109, 066803 (2012).

${ }^{23}$ H. Z. Lu, J. Shi, and S. Q. Shen, Phys. Rev. Lett. 107, 076801 (2011).

${ }^{24}$ H. T. He, G. Wang, T. Zhang, I. K. Sou, G. K. L. Wong, J. N. Wang, H. Z. Lu, S. Q. Shen, and F. C. Zhang, Phys. Rev. Lett. 106, 166805 (2011).

${ }^{25}$ D. Culcer, E. H. Hwang, T. D. Stanescu, and S. Das Sarma, Phys. Rev. B 82, 155457 (2010).

${ }^{26}$ V. Gurarie, Phys. Rev. B 83, 085426 (2011).

${ }^{27}$ G. Tkachov and E. M. Hankiewicz, Phys. Rev. B 84, 035444 (2011).

${ }^{28}$ C. M. Wang and F. J. Yu, Phys. Rev. B 84, 155440 (2011).

${ }^{29}$ H. K. Pal, V. I. Yudson, and D. L. Maslov, Phys. Rev. B 85, 085439 (2012).

${ }^{30}$ Z. Li and J. P. Carbotte, Phys. Rev. B 87, 155416 (2013).

${ }^{31}$ J. B. Pendry and D. R. Smith, Phys. Today 57, 37 (2004).

${ }^{32}$ D. R. Smith, J. B. Pendry, and M. C. K. Wiltshire, Science 305, 788 (2004).

${ }^{33}$ L. A. Falkovsky and S. S. Pershoguba, Phys. Rev. B 76, 153410 (2007).

${ }^{34}$ W. K. Tse and A. H. MacDonald, Phys. Rev. B 84, 205327 (2011).

${ }^{35}$ M. C. Chang and M. F. Yang, Phys. Rev. B 80, 113304 (2009).

${ }^{36}$ F. Wilczek, Phys. Rev. Lett. 58, 1799 (1987).

${ }^{37}$ J. D. Jackson, Classical Electrodynamics, 3rd ed. (Wiley, New York, 1999).

${ }^{38}$ O. Hosten and P. Kwiat, Science 319, 787 (2008). 